\title{
Expression of chicken interleukin- 2 by a highly virulent strain of Newcastle disease virus leads to decreased systemic viral load but does not significantly affect mortality in chickens
}

Leonardo Susta ${ }^{1,4}$, Diego G. Diel ${ }^{1,5}$, Sean Courtney ${ }^{1}$, Stivalis Cardenas-Garcia ${ }^{1}$, Roy S. Sundick ${ }^{3}$, Patti J. Miller ${ }^{1}$, Corrie C. Brown ${ }^{2}$ and Claudio L. Afonso ${ }^{1 *}$

\begin{abstract}
Background: In mammals, interleukin 2 (IL-2) has been shown to decrease replication or attenuate pathogenicity of numerous viral pathogens (herpes simplex virus, vaccinia virus, human respiratory syncytial virus, human immunodeficiency virus) by activating natural killer cells (NK), cytotoxic T lymphocytes and expanding subsets of memory cells. In chickens, IL-2 has been shown to activate T cells, and as such it might have the potential to affect replication and pathogenesis of Newcastle disease virus (NDV).

Methods: To assess the effect of IL-2 during NDV infection in chickens, we produced a recombinant virulent NDV strain expressing chicken IL-2 (rZJ1-IL2). The effects of IL-2 expression were investigated in vivo using the intracerebral pathogenicity index (ICPI) in day-old chicks and pathogenesis experiments in 4-week-old chickens. In these studies, rZJ1-IL2 was compared to a control virus expressing the green fluorescent protein (rZJ1-GFP).

Assessed parameters included survival curves, detailed histological and immunohistochemical grading of lesions in multiple organs, and virus isolation in blood, spleen and mucosal secretions of infected birds.

Results: At the site of infection (eyelid), expression of IL-2 was demonstrated in areas of rZJ-IL2 replication, confirming IL-2 production in vivo. Compared to rZJ1-GFP strain, rZJ1-IL2 caused milder lesions and displayed decreased viral load in blood, spleen and mucosal secretions of infected birds. In the rZJ1-IL2-infected group, virus level in the blood peaked at day 4 post-infection (pi) $\left(10^{3.46} \mathrm{EID}_{50} / 0.1 \mathrm{ml}\right)$ and drastically decreased at day $5 \mathrm{pi}$ $\left(10^{0.9} \mathrm{EID}_{50} / 0.1 \mathrm{ml}\right)$, while in the $\mathrm{rZJ1}$-GFP-infected group virus levels in the blood reached $10^{5.35} \mathrm{EID}_{50} / 0.1 \mathrm{ml}$ at day 5. However, rZJ1-IL2-infected groups presented survival curves similar to control birds infected with rZJ1-GFP, with comparable clinical signs and $100 \%$ mortality. Further, expression of IL-2 did not significantly affect the ICPI scores, compared to rZJ1-GFP strain.
\end{abstract}

Conclusions: Increased expression of chicken IL-2 during virulent NDV replication in naïve chickens decreased viral titers in blood, spleens, oral and cloacal secretions on day 4-5 post infection. This is consistent with the previously described role of IL-2 in enhancing the clearance of viruses in mammals, such as human respiratory syncytial virus.

\footnotetext{
* Correspondence: Claudio.Afonso@ars.usda.gov

'USDA ARS, Southeast Poultry Research Laboratory, 934 College Station Rd,

Athens, GA 30605, USA

Full list of author information is available at the end of the article
} reproduction in any medium, provided you give appropriate credit to the original author(s) and the source, provide a link to the Creative Commons license, and indicate if changes were made. The Creative Commons Public Domain Dedication waiver (http://creativecommons.org/publicdomain/zero/1.0/) applies to the data made available in this article, unless otherwise stated. 


\section{Background}

Newcastle disease (ND) severely affects poultry worldwide and causes severe economic losses both in developing and developed countries [1]. The disease is caused by virulent strains of Newcastle disease virus (NDV), an enveloped virus classified in the Mononegavirales order, Paramyxoviridae family, Avulavirus genus [2]. NDV strains have a non-segmented, negative sense RNA genome of approximately $15.2 \mathrm{~kb}$, which encodes for at least seven proteins, namely nucleoprotein (NP), phosphoprotein $(\mathrm{P}), \mathrm{V}$ protein (transcribed by post-transcriptional modification of the $\mathrm{P}$ mRNA), matrix (M), hemagglutinin neuraminidase (HN), fusion (F), and the large polymerase (L) [3]. According to international standards, NDV strains can be classified as virulent or non-virulent, based on the intracerebral pathogenicity index (ICPI), and the amino acid sequence of the F protein [4]. A NDV strain is considered virulent if it has an ICPI greater than or equal to 0.7 , or if the F protein has at least three basic amino acids from residues 113 to 116 and a phenylalanine at position 117 [3-5].

The ability to cause clinical signs and lesions varies greatly among NDV strains [6]. Clinically, NDV strains have been classified as highly virulent (velogenic), moderately virulent (mesogenic), and non-virulent (lentogenic and asymptomatic enteric strains). Velogenic strains are divided into viscerotropic, if they cause severe necro-hemorrhagic lesions in visceral organs, or neurotropic if they cause mainly neurological disease $[3,6]$.

Based on transcriptional analysis studies, infection with virulent strains of NDV induces transcriptional upregulation of numerous cytokines, such as interferon alpha (IFN- $\alpha$ ), interferon gamma (IFN- $\gamma$ ), interleukin 8 (IL-8), and interleukin 2 (IL-2) [7-10]. It is speculated that these cytokines might modulate the immune response during infection. It remains unclear, however, if their expression contributes to NDVinduced tissue damage and mortality (similar to the "cytokine storm" seen in mammals [11-14]), or represents an inadequate response to control virus replication and limit tissue damage.

In mammals, IL-2 facilitates clearance and decreases pathogenicity of numerous viral pathogens. In mice, a recombinant human herpes simplex-1 virus (HSV-1) expressing IL-2 showed decreased mortality compared to the wild-type parental strain, and this protection was linked to the activity of effector CD4+ and CD8+ lymphocytes [15]. Similarly, a IL-2 double knock-out mice inoculated with HSV-1 in the conjunctiva showed higher mortality, increased magnitude of virus replication in the eye, and more pronounced blepharitis compared to IL-2competent mice [16]. Treatment of BALB/c mice with recombinant IL-2 decreased replication of murine cytomegalovirus (CMV) in vivo [17]. When cloned into the genome of the murine poxvirus (Ectromelia virus), expressed IL-2 was able to decrease the pathogenicity of this virus in athymic nude mice, most likely by inducing production of IFN- $\gamma$ by natural killer (NK) cells $[18,19]$. Most significantly, expression of IL-2 by the respiratory paramyxovirus human respiratory syncytial virus (hRSV) increased virus clearance in mice [20]. In humans, IL-2 was associated with non-cytolytic clearance of human immunodeficiency virus (HIV) in CD4+ cells [21].

Chicken IL-2 was first cloned in 1997 [22, 23]. In avian species, IL-2 has effects comparable to mammals, including lymphocyte proliferation, activation of NK cells, and clearance of intracellular pathogens [24, 25]. Moderate upregulation of IL-2 has been observed in tissues of chickens infected with virulent NDV strains [8-10], however little is known about the effect of IL-2 on the virulence of NDV, and it remains unclear if IL-2 might have an antiviral effect against this virus. Given the demonstrated antiviral effects of IL-2 against several mammalian viruses, and the pleiotropic effects that IL-2 has in the immune response (some of which have antiviral activity, such as promoting a Th1biased immune response and cell-mediated immunity), it is possible that IL-2 might contribute to a faster clearance of NDV during infection. To investigate the hypothesis that IL-2 has an antiviral effect against NDV in vivo, we constructed a velogenic NDV that expresses chicken IL-2 during viral replication, and we evaluated its effect in 4week-old chickens.

\section{Results}

\section{Production and rescue of recombinant viruses}

The full-length clone (FLC) plasmid of the virulent wild-type ZJ1 NDV strain was used to introduce the open-reading frame (ORF) of chicken IL-2, between the $\mathrm{P}$ and $\mathrm{M}$ genes, as previously described [26]. As a control for the increased genome size, the ORF of the enzymatically inactive green fluorescent protein (GFP) was inserted in the FLC plasmid at the same genomic location. In order to rescue the recombinant viruses, FLC plasmids containing the ORF of IL-2, GFP, or no insert were transfected in Hep-2 cells together with helper plasmids (NP, P and L genes from the NDV LaSota strain) in order to rescue rZJ1-IL2, rZJ1-GFP, and rZJ1, respectively. Recombinant viruses were successfully rescued in Hep- 2 cells and propagated with two to three passages in 10-day old specific pathogen free (SPF) embryonated chicken eggs. RNA was extracted from the allantoic fluid of infected eggs, and to confirm the identity of the virus, the region corresponding to the insertion site (nucleotides 2857-3676 of the rZJ1 genome) was amplified by reverse transcriptase PCR (RT-PCR) and sequenced to assess the presence of the inserted sequences (see Material and Methods). 


\section{High levels of IL2 are produced by rZJ1-IL2 in both in vitro and in vivo}

Concurrent expression of IL-2 and NDV NP was assessed both in vitro (DF-1 cells) and in vivo (embryonated eggs) by western blot (WB) (see Material and Methods). Immortalized chicken embryo fibroblasts (DF-1 cells) and 9 to 10-day embryonated SPF chicken eggs were infected with rZJ1-IL2, rZJ1-GFP, or simple media (control). At $48 \mathrm{~h}$ post-infection (hpi), cell supernatant, cells lysate, and allantoic fluid were harvested and assayed using WB. A band of approximately $15 \mathrm{KDa}$, corresponding to the molecular weight of chicken IL-2 [24] and possibly the glycosylated form of IL2, were detected from the supernatant and cell extracts of DF-1 cells infected with rZJ1-IL2, but not rZJ1-GFP, or in the control group (Fig. 1a and b). A band of approximately $53 \mathrm{KDa}$, corresponding to the predicted molecular weight of NDV NP [27], was detected from the supernatant and cell extracts of DF-1 cells infected with rZJ1-IL2 and rZJ1-GFP, but not in the control group (Fig. 1a and b). Similarly, strong bands of 15 $\mathrm{KDa}$ and $53 \mathrm{KDa}$ were observed from the allantoic fluid of rZJ1-IL2-infected eggs (Fig. 1b). Blots from allantoic fluid of rZJ1-GFP-infected eggs showed the $53 \mathrm{KDa}$ but no the $15 \mathrm{KDa}$ bands, whereas control eggs (non-infected) were negative for both bands (data not shown). Taken together these data show that, while both rZJ1-IL2 and rZJ1-GFP replicate in cell cultures and eggs, only rZJ1-IL2 expresses chicken IL-2 at high quantities both in vitro and in vivo.

\section{Expression of IL-2 does not affect NDV replication in DF-1 or HD-11 cells}

To assess the effect of chicken IL- 2 production on the growth characteristics of rZJ1-IL2 and to verify that insertion of a foreign gene had not caused delay in rZJ1-IL2 growth, multi-step growth curves were carried out with rZJ1-IL2, rZJ1-GFP and rZJ1 in immortalized chicken embryo fibroblast (DF-1) and immortalized chicken macrophage cell lines (HD-11) (Fig. 2a and b). HD-11 cells were chosen because they may be biologically more relevant than DF-1 cells, due to the preferential tropism of virulent NDV strains for mononuclear phagocytic cells [6]. Briefly, cells were plated at $1 \times 10^{6}$ cells/well (DF-1) or $7 \times 10^{5}$ cells/ well (HD-11) into 6-well plates, and were infected the next day with each NDV strain at a MOI $=0.01$. Supernatant was collected at $0,6,12,24,36,48$, and $72 \mathrm{hpi}$. Virus titers were assessed by limiting dilutions in 96-well plates in DF-1 cells and expressed as tissue culture infectious dose $50 \%\left(\mathrm{TCID}_{50}\right)$, according to the Spearmann-Karber method [28]. Statistical analysis (Two-way ANOVA, and Tukey test for multiple comparisons) demonstrated that virus titers at each time point did not differ between viruses $(p=0.268$ and $p=0.1426$ for the effect of virus strain in DF-1 and HD-11 cells, respectively), indicating that rZJ1-IL2, rZJ1-GFP, and rZJ1 had comparable replication

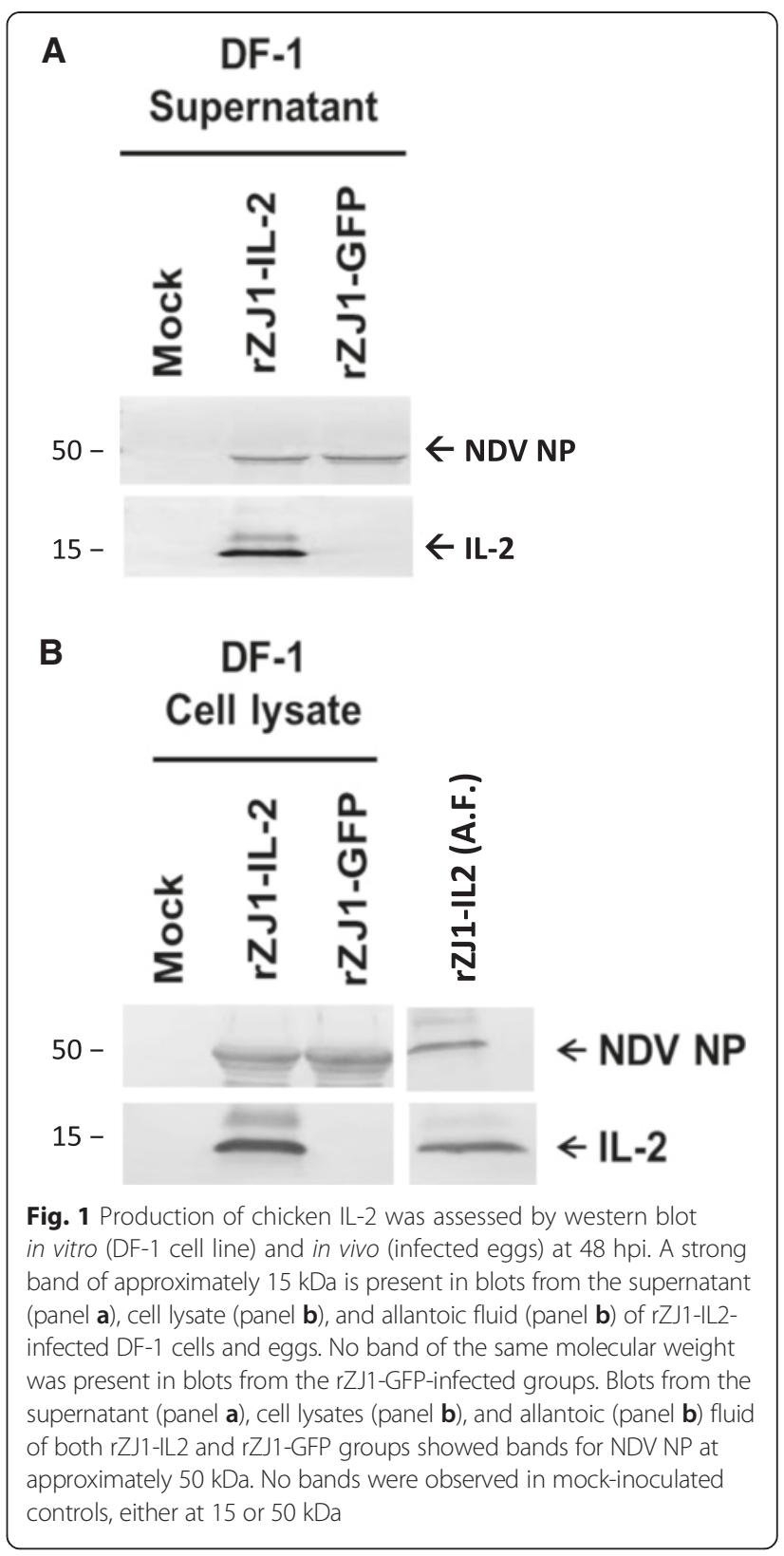

kinetics and that IL-2 expression did not affect replication of rZJ1 in DF-1 or HD-11 cells (Fig. 2a and b). In DF-1 cells, highest virus yields (mean $\pm \mathrm{SD}$, expressed as $\log _{10}$ $\mathrm{TCID}_{50}$ units / ml) were $7.63(+0.9,-0.11), 8.36(+0.4$, $-0.4)$, and $7.71(+0.18,-0.31)$ for rZJ1-IL2, rZJ1-GFP, and rZJ1 respectively (Fig. 2a). In HD-11 cells, highest yields were $7.87(+0.2,-0.4), 7.63(+0.09,-0.11)$, and 7.96 $(+0.05,-0.05)$ (Fig. 2b).

\section{ICPI}

The effect of IL2 expression on rZJ1 virulence was assessed in vivo using the intracerebral pathogenicity index (ICPI), which is the international standard to assess the virulence of NDV strains $[4,5]$. The ICPI score for 


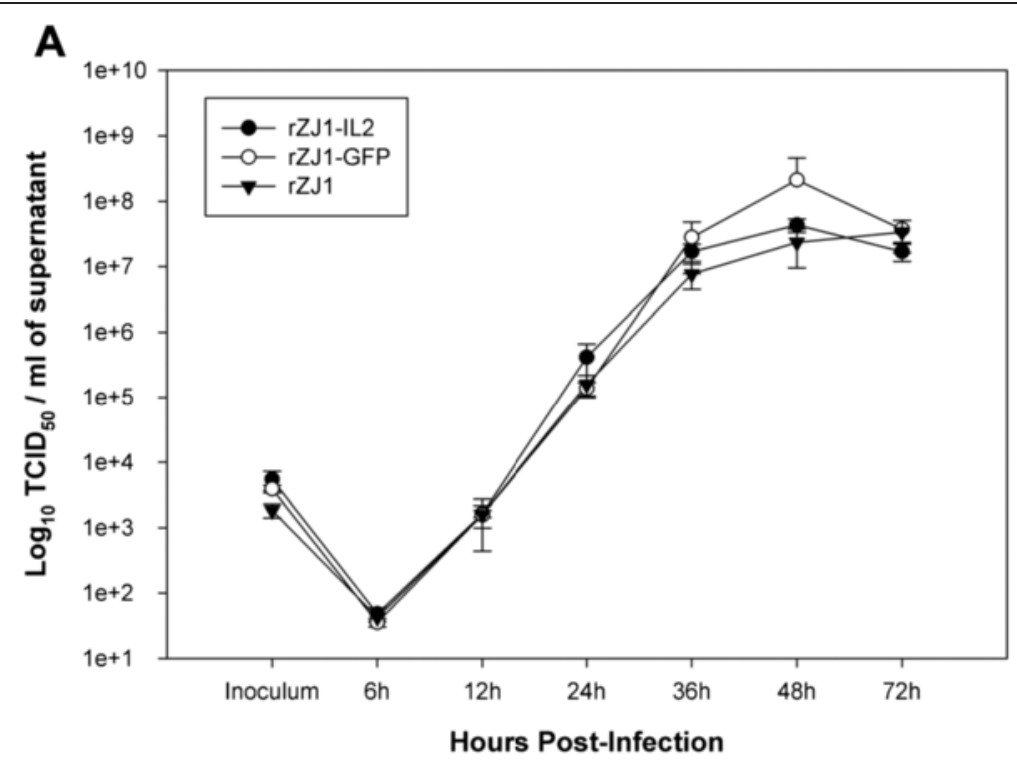

B

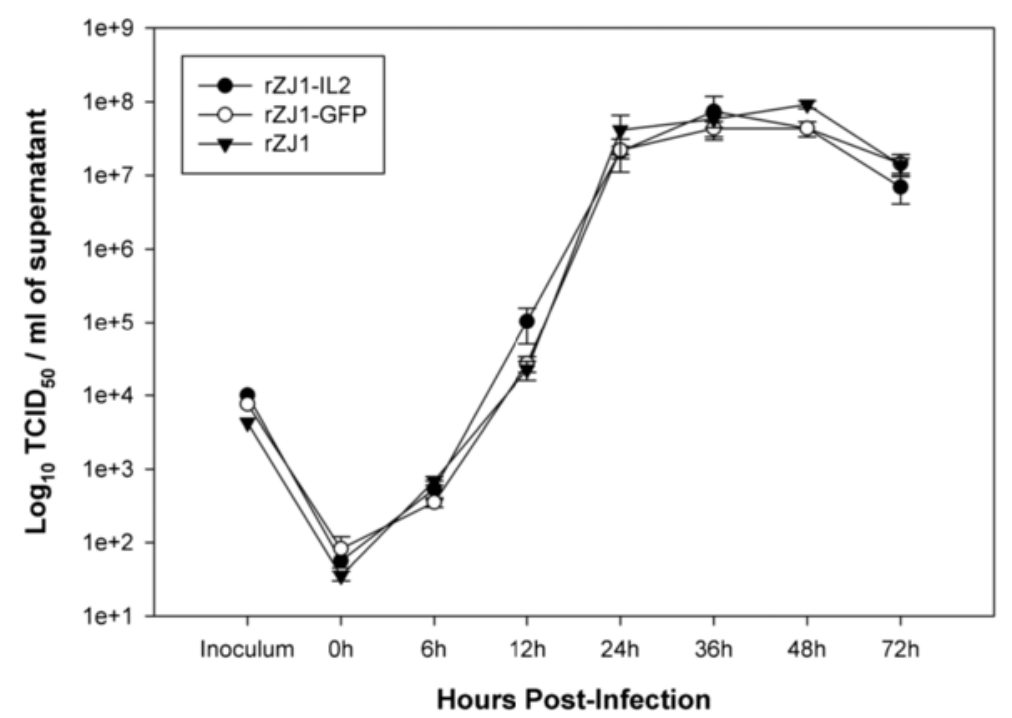

Fig. 2 Multi-step growth kinetics of rZJ1-IL2, rZJ1-GFP, and rZJ1 in immortalized chicken fibroblast cell line (DF-1, panel a), and immortalized chicken macrophage cell line (HD-11, panel b). DF-1 and HD-11 cells were infected with each virus at a MOI $=0.01$. At each time point, samples of supernatant were collected and titered by limiting dilutions in DF-1 cells (expressed as $T_{C I D} / \mathrm{ml}$ ). Results show that there are not significant differences (Two-way ANOVA) in the growth of the three viruses in either cell line. Bars represent standard error (SE), $n=3$

rZJ1-IL2 was 1.70, and the scores for rZJ1-GFP and rZJ1 have been previously published by our laboratory (using the same SPF White Leghorn flock) and are 1.64 and 1.85 [26]. These results indicate that IL-2 expression did not significantly change NDV virulence in day-old chickens, compared to the same virus expressing a gene with not known biological activity (GFP). Both rZJ1-IL2 and rZJ1-GFP have a lower ICPI score compared to the parental strain rZJ1 due to insertion of an additional transcriptional cassette $[29,30]$.

\section{Survival curves and clinical assessment}

Although the ICPI test is broadly used to assess the pathogenicity of NDV strains, it is an artificial system in one day old chicks that does not always accurately predict the lesion-inducing ability of strains inoculated through a natural route of infection, nor it is comprehensive enough to estimate minor differences in virus replication or clinical signs in adult birds [6]. For this purpose, groups of 4-week-old White Leghorn chickens were inoculated (eyelid instillation) with rZJ1-IL2 or 
rZJ1-GFP at three different target doses: $10^{4}$ (low), $10^{5.5}$ (medium), $10^{6.5}$ (high) TCID $_{50} /$ bird. Birds were monitored for clinical signs and deaths were recorded to produce a survival curve during 15 days (based on the expected time for all of the animals to die). Survival curves are presented in Fig. 3 and a detailed summary of the observed clinical signs is shown in Additional file 1: Table S1. Back-titers of virus inoculum were: $10^{4.38}$, $10^{5.88}$, and $10^{6.25} \mathrm{TCID}_{50} /$ bird for rZJ1-IL2, and $10^{3.75}$, $10^{5.5}$, and $10^{6.38} \mathrm{TCID}_{50} /$ bird for rZJ1-GFP.

Conjunctivitis was first observed at day 2 postinfection (pi) in birds infected with the high dose of rZJ1-IL2. Overall, conjunctivitis was more pronounced and severe in the rZJ1-IL2-infected birds than in the rZJ1-GFP-infected groups (Fig. 4, panel a). At day 4 and 5 pi, up to $13 \%$ of the rZJ1-IL2-infected birds developed remarkably severe comb hemorrhages and edema (low and medium dose) (Fig. 4, panel b). Both in rZJ1-IL2 and rZJ1-GFP groups, birds developed depression and neurological signs by day 4 pi. Neurological signs consisted of head twitch, inability to stand, or hypermetric gait. Death patterns were as follows: for the low dose inoculation group, all rZJ1-IL2 and rZJ1-GFP-infected birds died at day 11 and $9 \mathrm{pi}$, respectively; for the medium dose inoculation group, all rZJ1-IL2 and rZJ1-
GFP-infected birds died at day $7 \mathrm{pi}$; and for the high dose inoculation group, all rZJ1-IL2 and rZJ1-GFPinfected birds died at day 7 and 5 pi, respectively. Birds infected with rZJ1-IL2 took a longer time ( $48 \mathrm{~h}$ ) overall to cause death of all the infected birds compared to rZJ1-GFP in the low and high dose infection groups, suggesting a protective effect of IL2, however no statistical differences in mortality (log-rank test, multiple comparisons) were observed between groups inoculated with the same virus concentrations (Fig. 3). Mock-infected birds showed no mortality or clinical signs.

\section{Pathogenesis experiment}

A second animal experiment was conducted in order to assess in more details the ability of rZJ1-IL2 to cause lesions, and to evaluate its tissue distribution in key organs. To evaluate early development of gross and microscopic lesions, samples were taken during the first 5 days of infection. Groups of 20, 4-week-old chickens were inoculated (eyelid instillation) with either rZJ1-IL2 or rZJ1-GFP at a dose of $10^{5.6}$ and $10^{6.2} \mathrm{TCID}_{50} / \mathrm{bird}$, respectively, as assessed by backtitration (target dose $10^{5.5}$ ). A BHI-inoculated group served as mock control. Birds were examined daily and each day 5 animals were

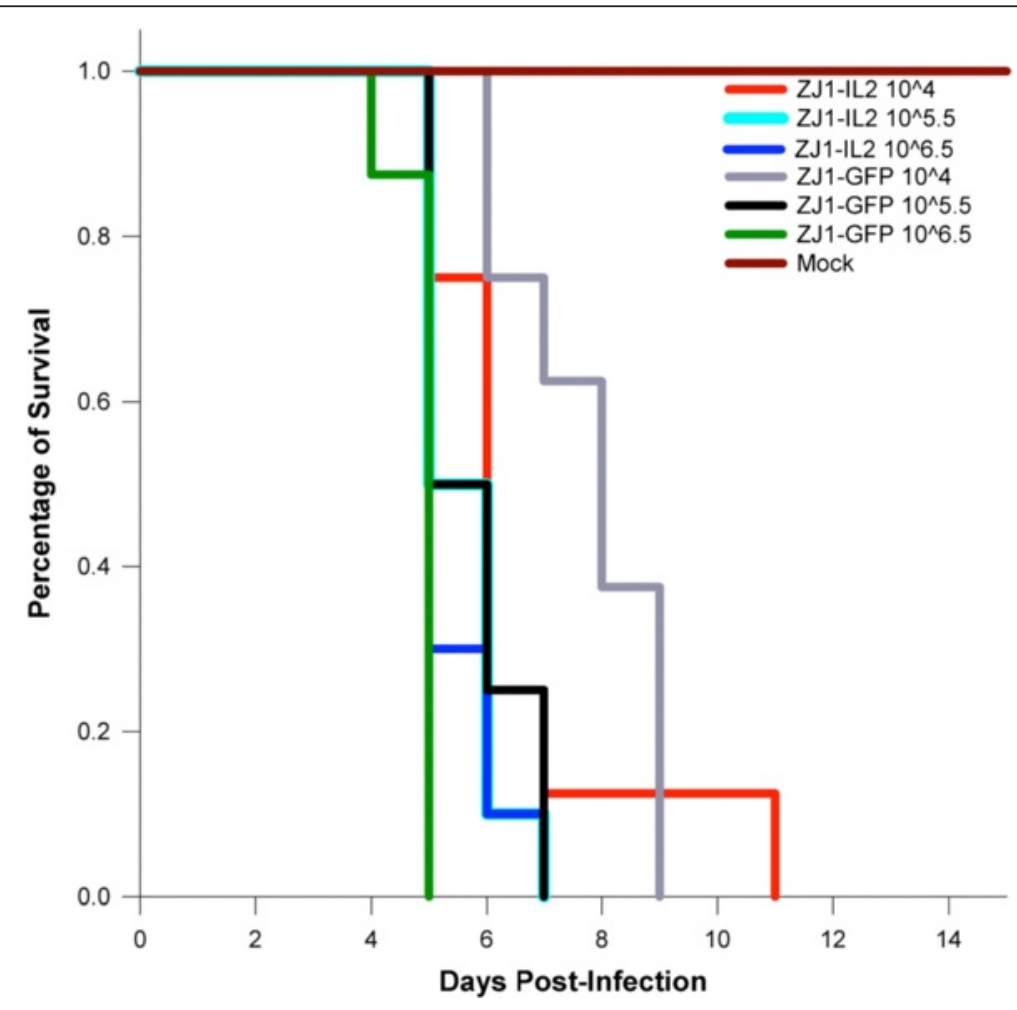

Fig. 3 Survival curves in 4-week-old White Leghorn chickens illustrating mortality rates between rZJ1-IL2 and rZJ1-GFP-infected groups at three different doses of inoculum (low, $10^{4} \mathrm{TCID}_{50} /$ bird; medium, $10^{5.5} \mathrm{TCID}_{50} /$ bird; high, $10^{6.5} \mathrm{TCID}_{50} /$ bird). Each color represents a different group at a different inoculum amount. No statistical differences were observed between rZJ1-IL2 or rZJ1-GFP groups within the same inoculum dose (log-rank test) 


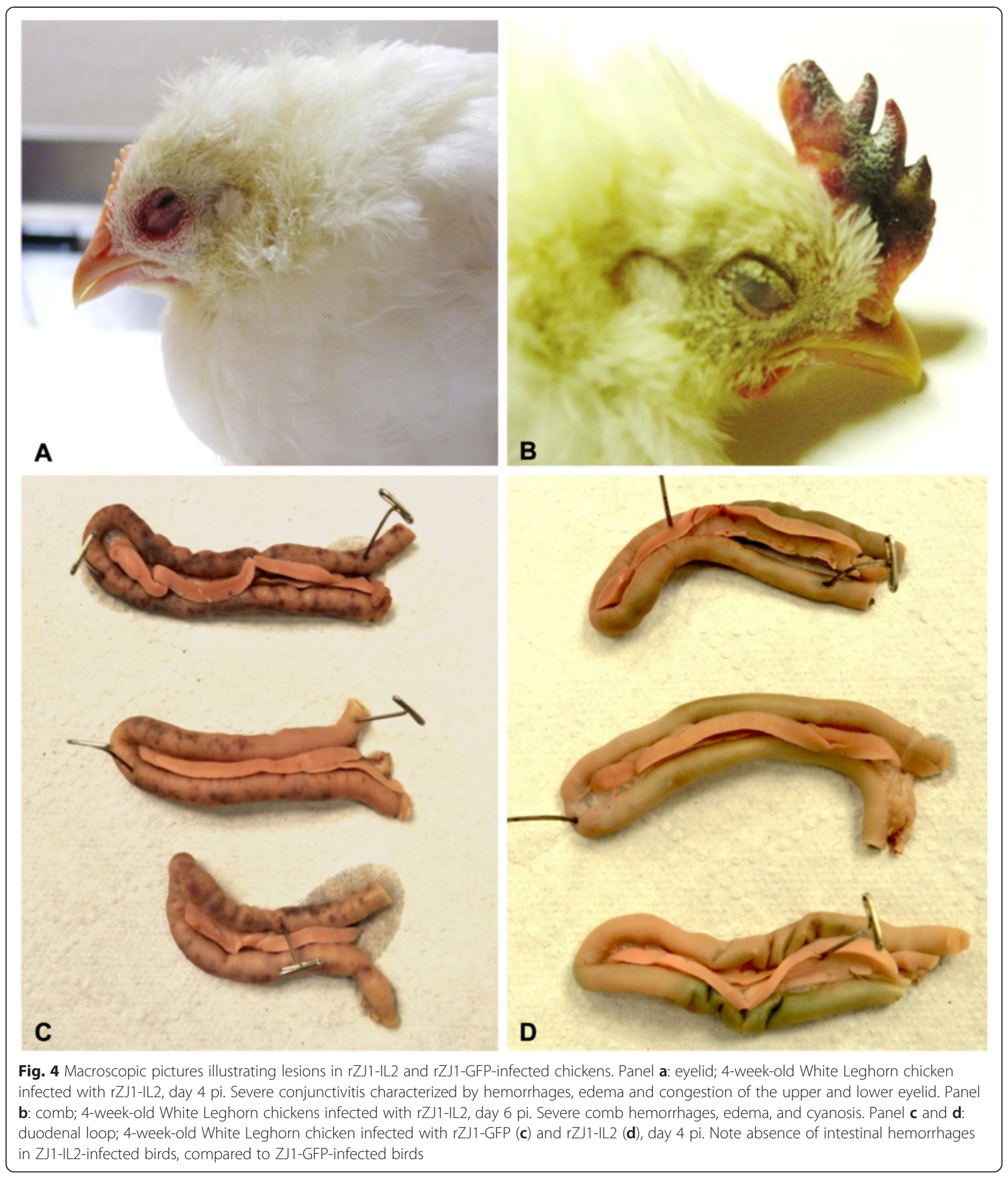

used to collect swabs (oral and cloacal), blood, and spleens for virus isolation (VI) and titration. Three animals were necropsied at each time point for assessment of gross lesions and sampling of tissues for histopathology. Detailed summary of clinical signs and gross lesions is reported in Table 1.
Clinical signs and mortality for each infection group are summarized in Table 1. In both groups, clinical signs consisted of various degrees of conjunctivitis and depression, which worsened until day 5 pi (end of the experiment). Similar to what was observed in the survival experiment, birds infected with rZJ1-IL2 displayed moderate to severe 
Table 1 Summary of clinical signs and lesions in 4-week-old chickens infected with rZJ1-GFP, rZJ1-IL2, and BHI

\begin{tabular}{ll}
$\begin{array}{l}\text { Recombinant } \\
\text { virus }\end{array}$ & Clinical signs \\
\hline rZJ1-GFP & Day 1 pi: no signs \\
& Day 2 pi: no signs \\
& Day 3: mild conjunctivitis \\
& \\
& Day 4 pi: moderate conjunctivitis and depression (3/10 had \\
& severe depression) \\
& Day 5 pi: severe conjunctivitis, severe depression: $3 / 5$ were \\
unable to stand; $1 / 5$ had neurological signs and depression, 1/5 \\
died overnight. All birds were euthanized in extremis. \\
Day 1 pi: no signs \\
rZJ1-IL2 & Day 2 pi: moderate conjunctivitis
\end{tabular}

Day 3 pi: moderate conjunctivitis and mild depression Day 4 pi: moderate to severe conjunctivitis, depression, and one
animal (1/10) with neurological signs (unable to stand with head
twitch)

Day 5 pi: bilateral moderate to severe conjunctivitis, depression

\section{Lesions}

Day 2 pi: splenomegaly (3/3); petechial hemorrhages in thymus $(2 / 3)$.

Day 3 pi: conjunctivitis (2/3); mottled and enlarged spleen (3/3).

Day 4 pi: conjunctivitis (1/3); thymic atrophy (2/3); enlarged mottled spleen (2/3); petechial hemorrhages diffuse in the intestine (3/3); necrosis in cecal tonsils (2/3); hemorrhages in proventriculus (1/3); bursal atrophy (1/3); severely dehydrated (1/3).

Day 5 pi: enlarged mottled spleen (3/3); hemorrhages in: duodenal loop, cecal tonsils, and throughout intestine (3/3); thymic atrophy (3/3); petechial hemorrhages in proventriculus (1/3).

Day 2 pi: conjunctivitis (2/3); splenomegaly (3/3); hemorrhages in: cecal tonsils (2/3), bursa (1/3), and thymus (1/3).

Day 3 pi: conjunctivitis (3/3); mottled and enlarged spleen (3/3); diarrhea (1/3).

Day 4 pi: severe bilateral conjunctivitis (3/3); enlarged and mottled spleen (3/3); necrosis in cecal tonsils (2/3); mild thymic atrophy $(1 / 3)$.

Day 5 pi: conjunctivitis (2/3); bursal atrophy (3/3); thymic atrophy (1/3); enlarged and mottled spleen (1/3); mild hemorrhages in duodenal loop (1/3); cecal tonsils necrosis (1/3); severe dehydration (2/3).

$\mathrm{BHI}$

No lesions or clinical signs observed.

Numbers indicate the fraction of birds presenting a certain clinical sign or lesion over the number of birds remaining in the cage (clinical signs), or the number of birds necropsied that day (lesions)

conjunctivitis earlier (day 2 pi) than those infected with rZJ1-GFP (day 3 pi). Birds infected with rZJ1-IL2 showed moderate depression at day 4 and $5 \mathrm{pi}$; however, no bird was terminally ill at the end of the experiment (day 5 pi). In rZJ1-GFP-infected birds, overall demise and severe depression became evident starting at day $4 \mathrm{pi}$ and culminated at day 5 pi when one bird died spontaneously $(1 / 5)$ and the rest $(4 / 5)$ were euthanized in extremis.

Gross pathological findings for both experimental groups are presented in Table 1. Both rZJ1-IL2 and rZJ1GFP caused similar lesions, which targeted mostly lymphoid organs and consisted of conjunctivitis, atrophy and necrosis of thymus, spleen, cecal tonsils and bursa of Fabricius (Table 1). Main differences among viruses consisted in the severity and extent of multifocal petechial intestinal hemorrhages at day 4 and $5 \mathrm{pi}$, which were severe and observed in six rZJ1-GFP-infected birds (Fig. 4, panel c), and mild and observed in only two rZJ1-IL2-infected birds (Fig. 4, panel d). Birds in the mock-infected group did not present any clinical signs or macroscopic lesions.

\section{Histopathology}

Severity of histopathological lesions in selected organs is summarized in Table 2. Overall, lesions between the two groups had similar severity at day 2 and 3 pi, while significant differences became evident at day 4 and 5 pi, when lesions were markedly milder in rZJ1-IL2- compared to rZJ1-GFP-infected birds (Table 2). In both groups at days 2 and $3 \mathrm{pi}$, there was prominent conjunctivitis, with abundant infiltration of inflammatory cells and extensive edema that progressed at days 4 and $5 \mathrm{pi}$ displaying fibrin exudation and necrosis (Fig. 5, panel a, b). At day 2 and 3 pi, in both rZJ1-IL2- and rZJ1-GFPinfected birds, the spleen showed prominent macrophages compared to mock-infected birds, resulting in overall increased size of the Schweigger-Seidel sheaths (splenic ellipsoids), which appeared almost confluent.

At day 4 and 5 pi, rZJ1-IL2-infected birds displayed prominent and confluent Schweigger-Seidel (ellipsoids) sheaths, however no extensive lymphoid depletion or fibrin exudation was observed (Fig. 5, panel e). The cecal tonsils showed mild to moderate lymphoid depletion at day 4 and 5 pi. Bursa and thymus had more severe lesions, compared to the other lymphoid organs at day $5 \mathrm{pi}$, consisting of multifocal follicular lymphoid depletion in the bursa and severe diffuse lymphoid necrosis in the thymic cortex (prominent "starry sky" effect) associated with fibrin exudation (data not shown). By day 
Table 2 Intensity of lesions (HE) and immunohistochemical staining (IHC) for NDV nucleoprotein in selected organs by days post infection (dpi). BHI-infected group did not present any lesions

\begin{tabular}{|c|c|c|c|c|c|c|c|}
\hline \multirow[b]{2}{*}{ Organs } & \multirow[b]{2}{*}{ DPI } & \multicolumn{3}{|c|}{ rZJ1-GFP } & \multicolumn{3}{|c|}{ rZJ1-IL2 } \\
\hline & & 3 & 4 & 5 & 3 & 4 & 5 \\
\hline \multirow[t]{2}{*}{ Eyelid } & $\mathrm{HE}$ & +++ & +++ & + & +++ & +++ & +++ \\
\hline & $\mathrm{IHC}$ & ++ & +++ & ++ & ++ & ++ & + \\
\hline \multirow[t]{2}{*}{ Spleen } & $\mathrm{HE}$ & ++ & ++++ & +++++ & ++ & ++ & ++ \\
\hline & $\mathrm{IHC}$ & ++ & +++ & + & + & - & - \\
\hline \multirow[t]{2}{*}{ Thymus } & $\mathrm{HE}$ & - & +++ & +++ & - & + & +++ \\
\hline & $\mathbb{H C}$ & - & +++ & ++ & + & + & - \\
\hline \multirow[t]{2}{*}{ Bursa of Fabricius } & $\mathrm{HE}$ & + & +++ & +++ & + & +++ & +++ \\
\hline & $\mathrm{IHC}$ & + & +++ & ++ & - & + & - \\
\hline \multirow[t]{2}{*}{ Cecal Tonsils } & $\mathrm{HE}$ & + & +++ & +++ & + & ++ & + \\
\hline & $\mathbb{H C}$ & + & +++ & ++ & + & + & - \\
\hline \multirow[t]{2}{*}{ Lung } & $\mathrm{HE}$ & - & ++ & ++ & - & - & - \\
\hline & $\mathrm{IHC}$ & - & - & - & - & - & - \\
\hline \multirow[t]{2}{*}{ Heart } & $\mathrm{HE}$ & - & + & - & - & - & - \\
\hline & $\mathrm{IHC}$ & - & - & - & - & - & - \\
\hline \multirow[t]{2}{*}{ Brain } & $\mathrm{HE}$ & - & + & + & - & + & + \\
\hline & $I H C$ & - & - & - & - & - & - \\
\hline
\end{tabular}

\section{Histological grading}

Eyelid: + presence of severe edema, which expands the submucosa; ++ multifocal inflammatory infiltrate; +++ coalescing / diffuse inflammatory infiltrate

Thymus: lymphoid depletion and apoptosis / necrosis of the thymic cortex + $<20 \%$; ++ 26 to $50 \%$; +++> $50 \%$

Cecal tonsil and Bursa: lymphoid depletion / necrosis, $+<20 \%,++26$ to $50 \%$; $+++>50 \%$

Spleen: prominent macrophages surrounding the penicillary arteries, +; confluent Schweigger-Seidel sheaths, ++; lymphoid depletion and apoptosis + ++ ; diffuse necrosis and fibrin exudation ++++

Brain: vascular reactivity, + ; perivascular mononuclear cuffing ++ Immunohistochemical grading

- = no IHC signal present

$+=$ rare cells in the section are positive on $\mathrm{IHC}$

$++=$ positive cells seen, $<50 \%$ of all high power fields (HPF)

$+++=$ positive signal seen in $>50 \%$ of $\mathrm{HPF}$

4 pi, rZJ1-GFP-infected birds presented very severe lesions in all the lymphoid organs, consisting of lymphoid depletion, necrosis, and multifocal fibrin deposition with greatest prominence in cecal tonsils and spleens. By day 5 pi, lesions consisting of lymphoid depletion, necrosis, and fibrin exudation also involved thymus and bursa and became progressively more severe in the spleen (Fig. 5, panel f).

In the brains of rZJ1-IL2-infected birds, at both day 4 (2/3 birds) and day 5 (2/3 birds) pi, there was accumulation of mononuclear cells within the perivascular spaces of scattered vessels (perivascular cuffing), mainly in periventricular regions and cerebellar peduncles. In the brain, at both day 4 and 5 pi, most birds inoculated with rZJ1-GFP (4/6 birds) did not show perivascular cuffing, but showed scattered glial nodules in the brainstem and multifocal vascular reactivity (plumped endothelial cells associated with few scattered mononuclear inflammatory cells within the vascular wall) in the periventricular areas.

\section{Immunohistochemistry for NDV nucleoprotein}

To detect viral distribution in tissues, IHC for NDV nucleoprotein was performed on samples from rZJ1-IL2-, rZJ1-GFP-, and mock-infected birds. A summary of the distribution and intensity of immunohistochemical (IHC) staining for the NDV NP is presented in Table 2. Immunohistochemical labeling for the NDV NP was mainly confined to the lymphoid tissues. Considering all time points, birds inoculated with either virus had $5 / 5$ positive lymphoid organs. However, IHC signal was markedly less intense and confined to few areas in the lymphoid organs of rZJ1-IL2- compared to rZJ1-GFPinfected birds, especially at day 4 and 5 pi. Immunolabelling for NDV NP was decreased in intensity in the eyelids of rZJ1-IL2- compared to rZJ1-GFP-infected birds, albeit lesions were comparable in severity (Fig. 5, panels $\mathrm{c}$ and $\mathrm{d}$ ). Differences in immunolabelling were striking in the spleen, where minimal to no signal of NDV NP was observed in rZJ1-IL2-compared to rZJ1GFP-infected birds (Fig. 5, panels g and h). Mockinfected birds did not present any immunoreactivity for NDV nucleoprotein.

\section{Co-expression of NDV NP and IL-2}

Sections of eyelid, which showed highest level of NDV replication by IHC, were chosen to assess IL-2 production by using IHC against chicken IL-2. Eyelids from rZJ1-IL2- and rZJ1-GFP-infected birds at day 4 and 5 pi were assayed for IHC for both NDV NP (Fig. 6, panels a, c, e) and chicken IL-2 (Fig. 6, panels b, d, f). Results showed that the all the eyelids of rZJ1-IL2-infected birds showed positive staining for IL-2 and NDV NP, while the eyelids of rZJ1-GFP-infected were negative for IL-2 and positive for NDV NP. In consecutive sections, NDV NP and IL-2 expression could be observed in the same areas, and occasionally in the same cells (in Fig. 6, compare panels a with $b$ and $c$ with $d$ ). These results show that IL-2 is expressed in the context of rZJ1-IL2 virus replication in vivo.

\section{Virus isolation and titration}

Systemic virus replication in infected birds was assessed by virus isolation and titration in whole blood (Fig. 7, panel a), spleens (Fig. 7, panel b), and in oral and cloacal secretions of rZJ1-IL2- and rZJ1-GFP-infected birds (Fig. 8). NDV was isolated via standard methods from tissues and swabs in embryonated chicken eggs, and titers were determined by limiting dilution and expressed as embryo infectious dose $50 \%\left(\mathrm{EID}_{50}\right)$ per $0.1 \mathrm{ml}$ [5]. Viremia from the rZJ1-IL2 group peaked at 

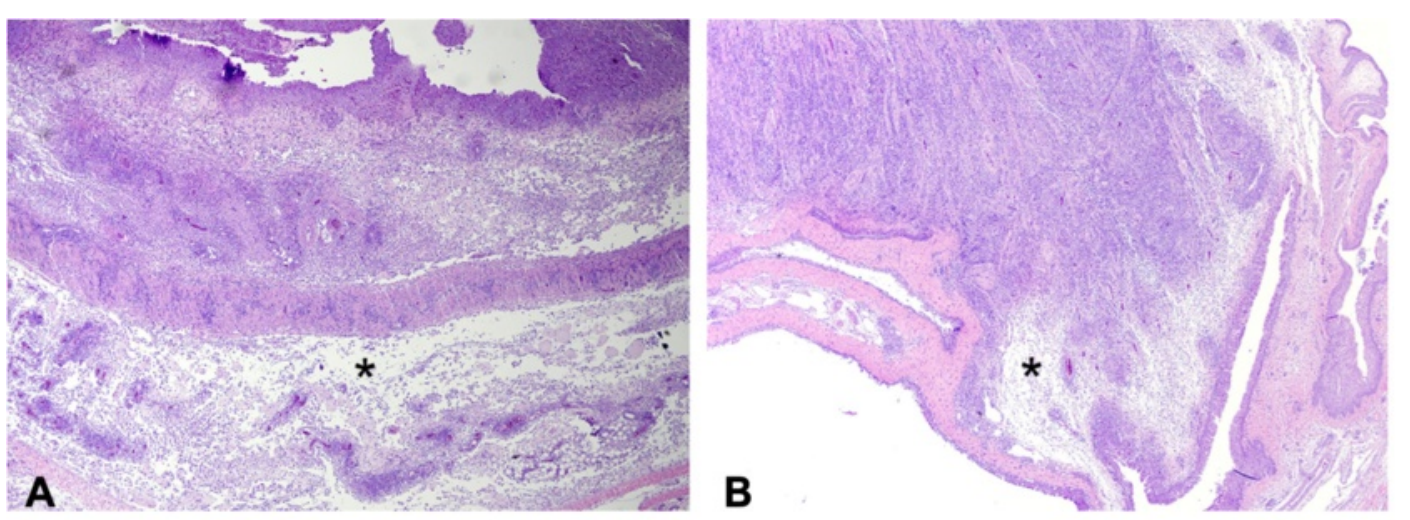

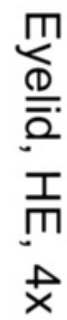

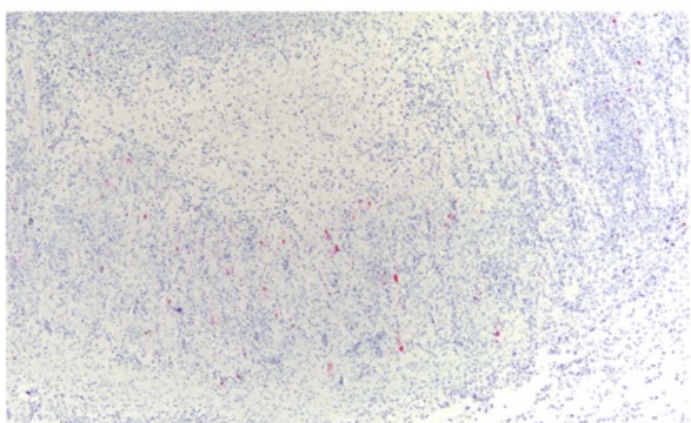

C
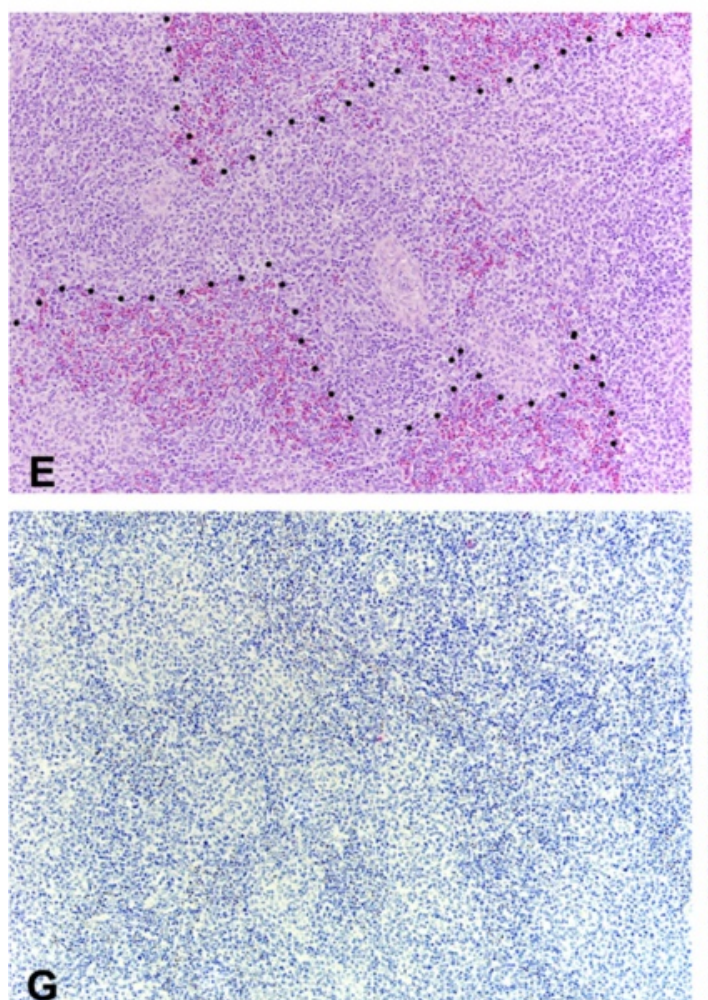

rZJ1-IL2-infected group
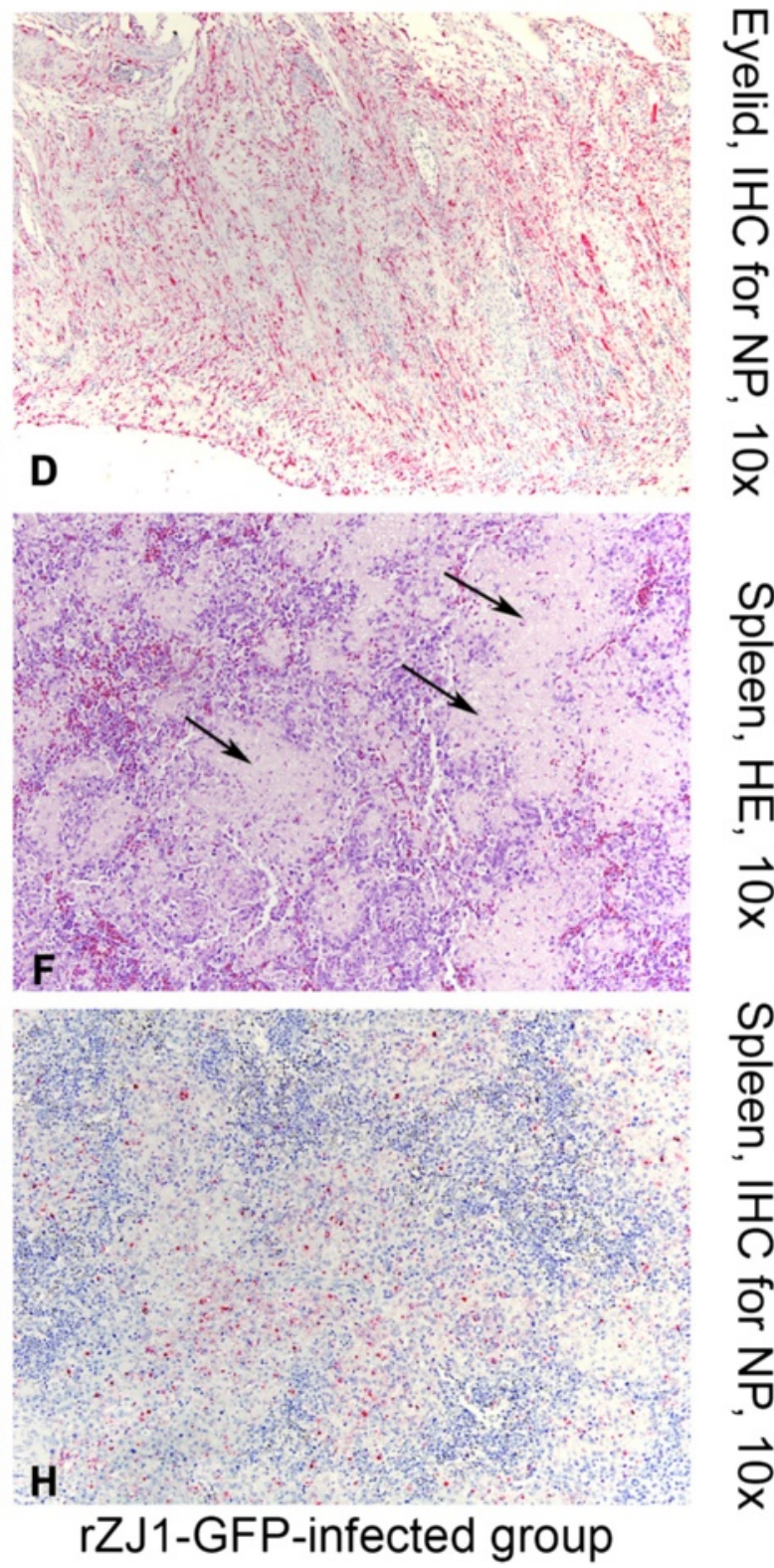

Fig. 5 (See legend on next page.) 
(See figure on previous page.)

Fig. 5 Photomicrographs illustrating hematoxylin and eosin staining (HE, first and third rows of panels) and immunohistochemistry (IHC, second and fourth rows of panels) on sections of eyelid (panels a-d) and spleen (e-f) at day 5 pi. Tissues were harvested from 4-week-old White Leghorn chickens infected with rZJ1-IL2 (first column of panels), and rZJ1-GFP (second column of panels). Alkaline phosphatase method and hematoxylin counterstain. In the eyelid, histopathological changes consist of severe edema (asterisks), which markedly expands the submucosa, accumulation of pleomorphic cellular infiltrate (macrophages, heterophils, lymphocytes), exudation of fibrin, and multifocal areas of coagulative necrosis. This severe conjunctivitis is similar in intensity in both rZJ1-II2 (panel a) and rZJ1-GFP (panel b) groups. Presence of lesion in the eyelid is associated with positive immunohistochemical labeling, which is intense and diffuse in rZJ1-GFP-infected birds (panel $\mathbf{d}$ ), while in rZJ1-IL2-infected birds is less intense and multifocal (panel c). In the spleen, rZJ1-IL2-infected birds display mild to moderate lymphoid depletion and accumulation of prominent macrophage in the ellipsoid areas, which appear confluent (dashed lines, panel e). These changes are associated with minimal NDV immunohistochemical labeling, which is not present at day 5 pi (panel g). Birds infected with rZJ1-GFP show severe lesions in the spleen, consisting of lymphoid depletion, prominent macrophages, exudation of fibrin and accumulation of necrotic debris (arrows, panel f). These lesions are associated with intense and diffuse immunohistochemical labeling for NDV (panel $\mathbf{h}$ )

day 4 pi (mean, $10^{3.46} \operatorname{EID}_{50}$ per $0.1 \mathrm{ml}$ of blood) and drastically decreased at day 5 pi (mean, $10^{0.9}$ EID $_{50}$ per $0.1 \mathrm{ml}$ of blood) (Fig. 7, panel a). In rZJ1-GFP-infected birds, viremia progressed from day 2 to day 5 pi when it reached $10^{5.35} \mathrm{EID}_{50}$ per $0.1 \mathrm{ml}$ of blood (mean). Differences in virus titers were determined as statistically significant between rZJ1-IL2 and rZJ1-GFP groups at day 4 and 5 pi (Mann-Whitney-Wilcoxon test, $p<0.05)$.

Similarly, the amount of virus present in the spleen of infected birds at days 4 and 5 pi was lower in the rZJ1IL2- than in rZJ1-GFP-infected birds. For rZJ1-IL2 and rZJ1-GFP groups respectively, at day $4 \mathrm{pi}$, titers in the spleens had an average of $10^{3.7}$ and $10^{4.99}$ EID $_{50}$ per $0.1 \mathrm{ml}$ of spleen homogenate, and at day $5 \mathrm{pi}$, titers were $10^{3.31}$ and $10^{4.60}$ (Fig. 6, panel b). Differences in virus titers were deemed statistically significant between rZJ1IL2 and rZJ1-GFP groups at day 4 pi (Mann-WhitneyWilcoxon test, $p<0.05$ ).

Results of titration of oral and cloacal secretions showed that rZJ1-IL2 shed in lower quantities compared to rZJ1-GFP at all time points tested (Fig. 8, panels a and b). Shedding in oral secretions for both rZJ1-IL2 and rZJ1-GFP peaked at day 4 pi (mean, rZJ1-IL2: $10^{5.21}$, rZJ1-GFP: $10^{6.18}$ EID $_{50}$ per $0.1 \mathrm{ml}$ ) and slightly decreased at day 5 pi (Fig. 8, panel a). In cloacal swabs, the highest amount of virus shed in the rZJ1-IL2 group was at day 5 pi (mean, $10^{3.62} \mathrm{EID}_{50} / 0.1 \mathrm{ml}$ ), while in the rZJ1-GFP group, the highest titer was at day 4 pi (mean, $10^{4.66}$ $\mathrm{EID}_{50} / 0.1 \mathrm{ml}$ ) (Fig. 8, panel b). Differences in virus titers were statistically significant between rZJ1-IL2 and rZJ1-GFP groups at day 5 pi for both oral and cloacal swabs (Mann-Whitney-Wilcoxon test, $p<0.05$ ).

\section{Discussion}

In the present study, the effects of the chicken IL-2 gene expression during in vivo infection of SPF chickens by a virulent NDV were investigated. A virulent NDV virus expressing IL-2 was compared to an identical clone expressing the GFP gene. Since it has been shown that the insertion of additional nucleic acids in the genome may slightly affect the capacity of the virus to replicate [29],
rZJ1-GFP was considered to be the most suitable control for rZJ1-IL2 in all animal experiments. This control has proven to be suitable for this experiment as no effect on viral replication was observed in highly controlled in vitro growth curves.

Animal experiments conducted in 4-week-old chickens demonstrated that expression of IL-2 by rZJ1-IL2 induced an attenuated phenotype compared to rZJ1GFP, resulting in decreased severity of hemorrhagic lesions in the intestine, and decreased severity of lymphoid depletion and necrosis in multiple lymphoid organs. Thus, as it would be expected by an effect produced on the host by a gene expressed during viral replication, these differences were more pronounced in tissues that are normally targeted by velogenic NDV [6], i.e., the lymphoid organs, which also have a higher numbers of possible effector cells that can be stimulated by IL- 2 .

The observed differences in pathology were accompanied with marked differences in the magnitude of tissue viral load (titers) between rZJ1-IL2- and rZJ1-GFPinfected birds. As shown by IHC, intensity of immunolabelling paralleled the severity and distribution of lesions, being less intense and less distributed in the organs of rZJ1-IL2- compared to rZJ1-GFP-infected birds.

The observed decreased viral load in different tissues suggests that IL-2 may have a general antiviral effect against NDV; however, the nature and extent of these effects await further investigation. The pleiotropic effects of IL-2 in mammals have been extensively reviewed [31], however, data in avian species are limited. In mammals, IL-2-dependent activation of effector cells has been proven important to control infection caused by viruses, such as HSV-1 [15, 16], cytomegalovirus [17], and lymphocytic choriomeningitis virus (in the persistent infection phase) [32]. Similar to what is described in the present study, there are several reports in the literature documenting attenuation of recombinant viruses expressing IL-2 in mice. For instance, a recombinant strain of HSV-1 expressing IL-2 was markedly attenuated in mice compared to wild-type parental strains, most likely due to activation of CD8+ and CD4+ effector T lymphocytes [15]; when expressed by a 

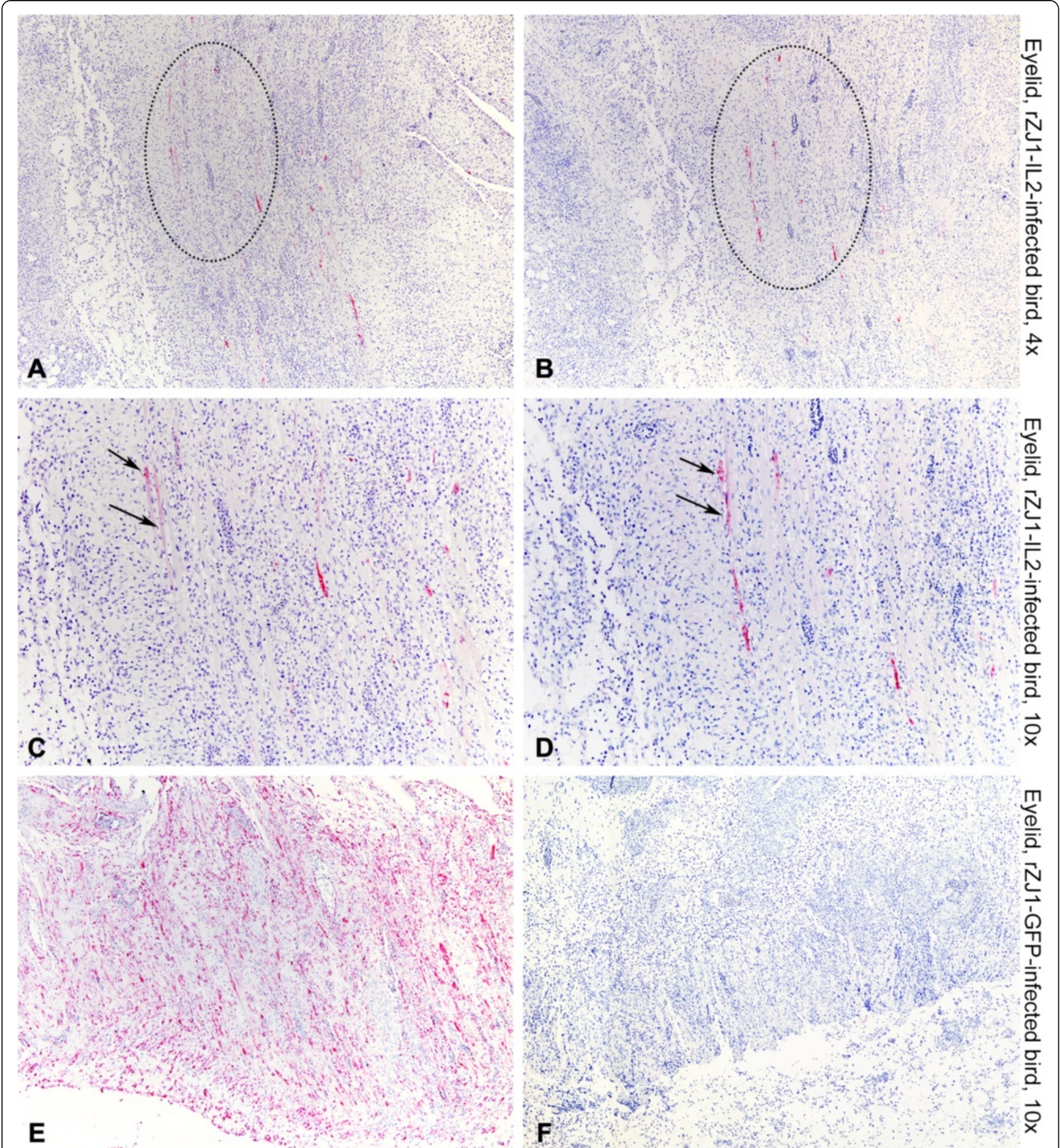

\section{IHC for NDV NP}

\section{IHC for chicken IL-2}

Fig. 6 Photomicrographs illustrating immunohistochemistry (IHC) for NDV NP (first column of panels) and IL-2 (second column of panels) on sections of eyelids. Tissues were harvested from 4-week-old White Leghorn chickens infected with rZJ1-IL2 (a-d) and rZJ1-GFP (e, f) at day 5 pi. Alkaline phosphatase method and hematoxylin counterstain. At low magnification, in the eyelids of rZJ1-IL2-infected birds, the same areas that are immunolabeled for NDV NP are also positive for chicken IL-2 (dotted circles). At higher magnification, scattered cells in consecutive sections are immunolabeled for both NDV NP and IL-2 (arrows). No signal for IL-2 is observed in sections of eyelids from rZJ1-GFP-infected animals (f) 


\section{A}

\section{Titers in blood}

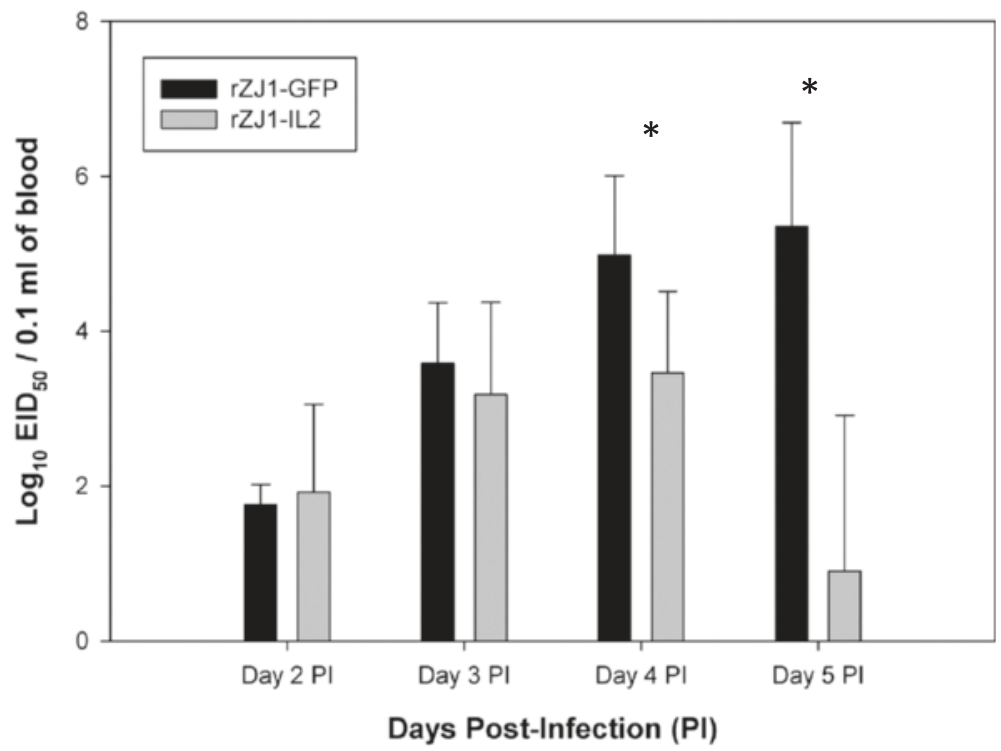

B

\section{Titers in spleen}

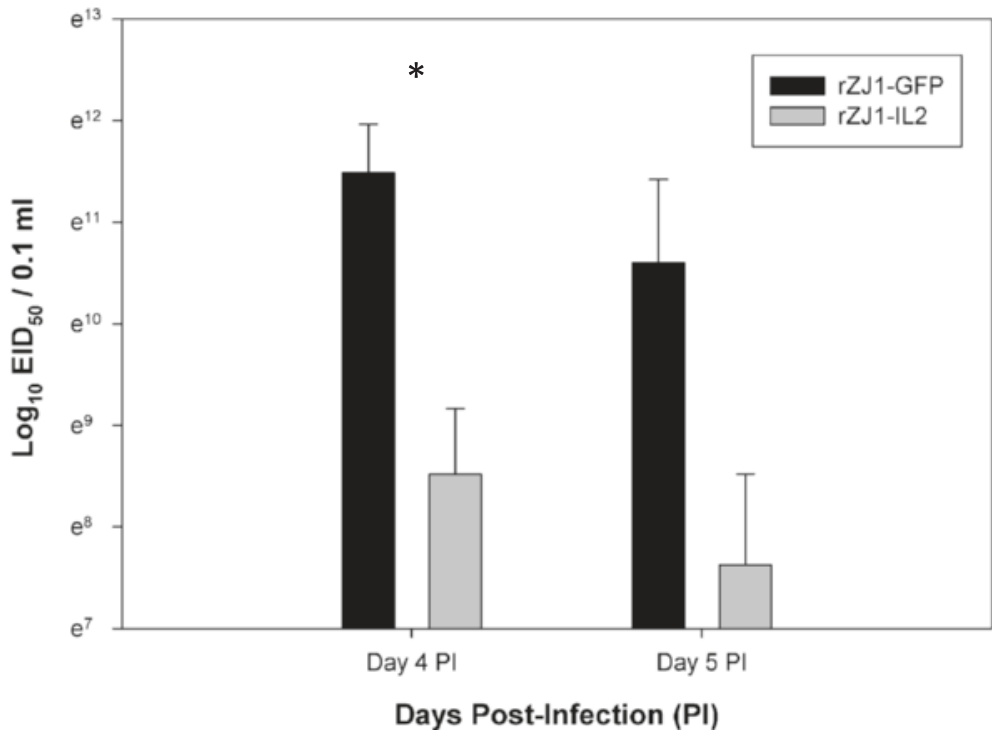

Fig. 7 Titration of rZJ1-GFP and rZJ1-IL2 in whole blood (panel a) and spleens (panel b) of infected birds. *indicates significant difference between rZJ1-IL2- versus rZJ1-GFP-infected groups $(p<0.05)$. Bars represent standard deviation, $n=5$

recombinant vaccinia virus, IL-2 was able to dramatically reduce morbidity and mortality in nude mice through upregulation and enhanced expression of IFN- $\gamma$ by NK cells $[18,19]$; a recombinant hRSV strain expressing IL-2 was moderately attenuated in respiratory tract of infected mice and induced upregulation of IFN- $\gamma$ in lung mononuclear cells [20].

Results of the present study showed that IL-2 attenuates the severity of lesions and virus distribution when expressed simultaneously with replication of a virulent NDV strain, suggesting a possible indirect antiviral effect of IL-2 against NDV. Similarly to the studies reported above, an antiviral effect of IL-2 against NDV could have been the direct consequence of the activation of effector cells, such as NK cells, or indirect production of more potent antiviral cytokines, such as IFN- $\gamma$. It is possible that a more pronounced effect of IL-2 gene may not have been detected in our study because of the highly 


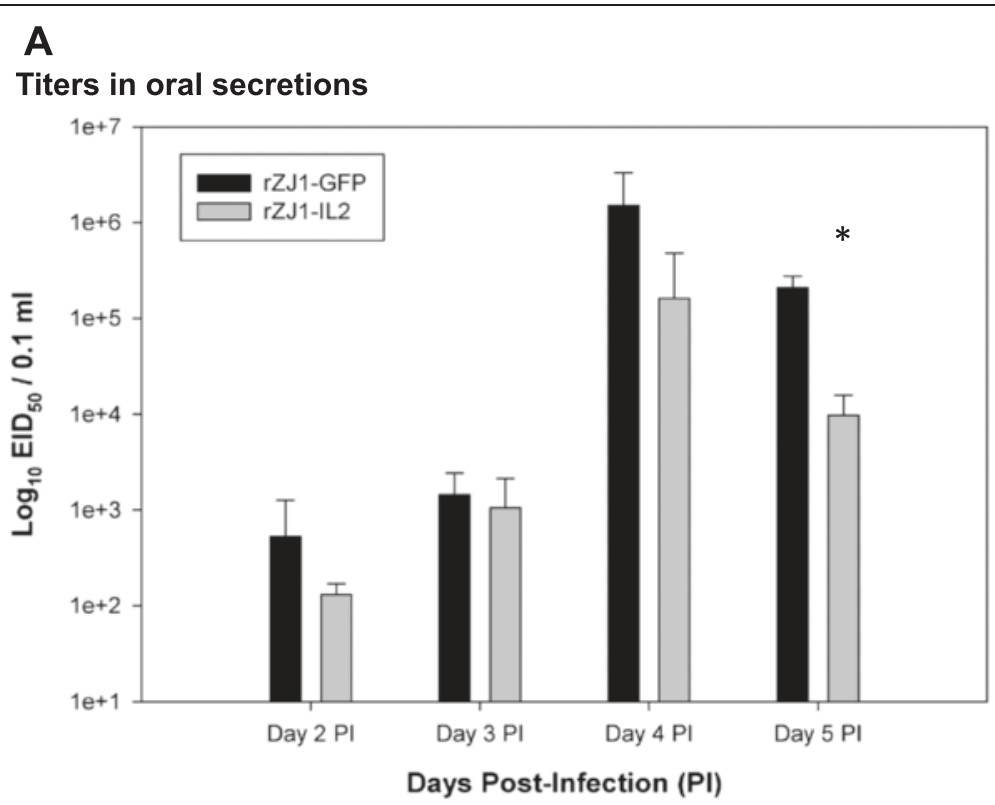

\section{B}

\section{Titers in cloacal secretions}

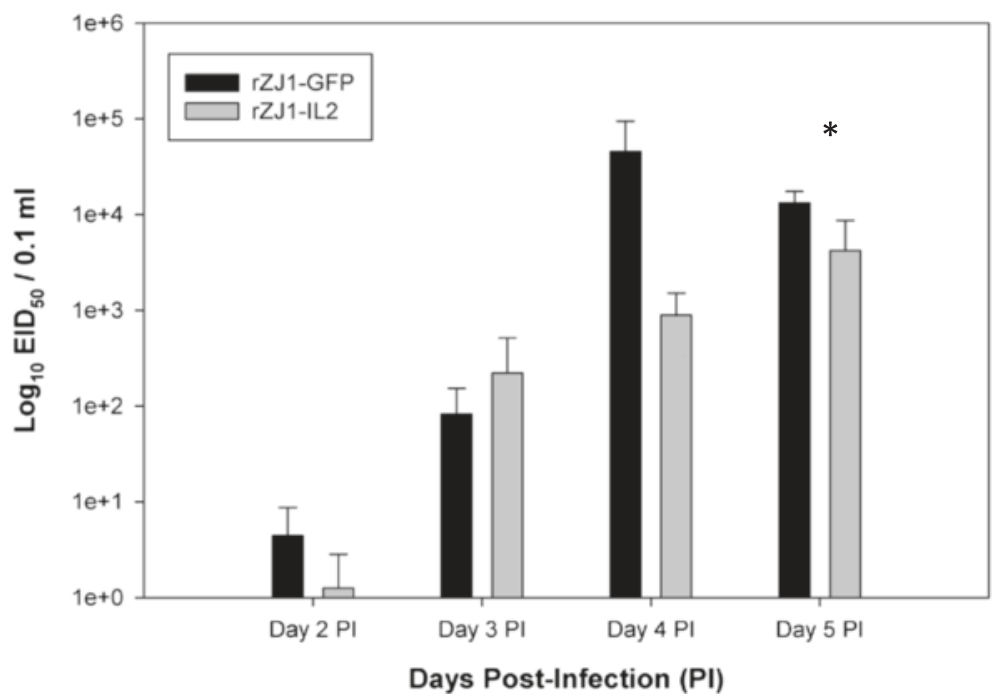

Fig. 8 Titration of rZJ1-IL2 and rZJ1-GFP in oral (panel a) and cloacal (panel b) secretions of infected birds. *indicates significant difference between rZJ1-GFP- versus rZJ1-IL2-infected groups $(p<0.05)$. Bars represent standard deviation, $n=5$

virulent characteristics of rZJ1 strain, which usually induces a disease course of only of a few days (usually up to $5[6])$. This timeframe may have been too short to yield a polarized Th1 response or to activate cytotoxic $\mathrm{T}$ cells (all functions of IL-2 [33]).

It is possible that the antiviral mechanisms observed in mammals could also develop in NDV-infected chickens, since in this species IL-2 has similar roles as in mammals. In fact, chicken IL-2 is produced by stimulated splenic lymphocytes, induces proliferation of lymphocytes [22, 24], contributes to differentiation/ activation of chicken regulatory $\mathrm{T}$ cells (T-regs) and NK cells [33,34], and is implicated in the development of cell-mediated immunity $[35,36]$. Cell-mediated immunity and NK cell activation, in turn, could avail NDV clearance [37]. It is possible that expression of IL-2 could have determined upregulation of other cytokines with more prominent antiviral activity, such as IFN- $\gamma$. For instance, in mice, IL- 2 is able to induce IFN- $\gamma$ production in peritoneal macrophages [38]. In a previous study, we have shown that expression of IFN$\gamma$ by the same NDV strain used in the present work 
(rZJ1-IFN $\gamma$ ), induced a dramatic decrease in morbidity and mortality of infected birds [26], suggesting that IFN- $\gamma$ has a more potent effect in controlling NDV infection than IL-2. Further studies are necessary to elucidate the transcriptional activity of chicken tissues infected with rZJ1-IL2.

\section{Conclusion}

In summary, our results show that production of IL-2 simultaneously with replication of a virulent NDV strain was sufficient to reduce systemic (blood) and localized (tissues) viral loads, however it was not sufficient to decrease mortality of infected chickens. These data are in agreement with observations made with other viral pathogens and suggest that IL-2 may work with other cytokines to control NDV infection in vivo. As it would be expected, the results suggest that expression of a single gene with antiviral properties may not be sufficient to substantially alter the outcome of infection caused by a highly virulent virus with a rapid disease course. Nonetheless, IL-2 partially decreased the pathogenicity of a velogenic NDV strain by lessening tissue damage and systemic viral load, suggesting that IL-2 in conjunction with other genes may have a greater attenuating effect with less-virulent NDV pathotypes (i.e., mesogenic or lentogenic strains), and therefore warranting further tests with these strains.

\section{Materials and methods}

\section{Cells and viruses}

DF-1 cells (Chicken embryo fibroblast cell line; ATCC CRL 12203) and HEp-2 cells (American Type Culture Collection, ATCC, Manassas, VA, CCL-23) were cultured in Dulbecco's modified Eagle's medium (DMEM) (with $5 \%$ fetal bovine serum (FBS) and $1 \%$ streptomycin / penicillin) at $37{ }^{\circ} \mathrm{C}$ with $5 \% \mathrm{CO}_{2}$. $\mathrm{HD}-11$ cells (chicken bone marrow macrophages cell line [39]) were cultured in Roswell Park Memorial Institute (RPMI) media (with $10 \%$ FBS with $1 \%$ streptomycin/penicillin at $37^{\circ} \mathrm{C}$ with $\left.5 \% \mathrm{CO}_{2}\right)$. The recombinant modified Vaccinia virus Ankara expressing the T7 RNA polymerase (a generous gift of Bernard Moss, National Institutes of Health) was grown in primary chicken embryo fibroblast cells. The recombinant NDV strains, rZJ1-GFP and rZJ1 were rescued from full-length clone plasmids as previously described [26, 29].

\section{IL-2 cloning}

The open reading frame (ORF) of chicken IL-2 cloned into pCR2.1 was a generous gift of Dr. Sundick (Wayne State University, MI). The IL-2 ORF was modified as follows: a Kozak sequence (GCCGCCACC) was added before the start codon (ATG); the nucleotide immediately after the first ATG codon was mutated from an adenosine to a guanidine residue $(A \rightarrow G)$, resulting in a substitution of a methionine to a valine in the amino acid sequence; a stretch of 6 histidine amino acids was added at the carboxy-terminal of the protein. This modified IL-2 ORF was previously shown to be expressed in eukaryotic systems and to be biologically active in chickens [22, 24]. For insertion within the rZJ1 genome, the "gene start" (GS), "gene end" (GE), and ApaI restriction site sequences were added to the IL-2 ORF by PCR amplification (High Fidelity PCR kit, Promega, Madison, WI) using primers IL2-F (5' -ctgggccctcttagaaaaaatacgggtagaagtaccggatccgccgccaccatgg- $\left.3^{\prime}\right)$ and IL2-R ( $5^{\prime}$-ggccggttgggccctctcctcaatgatgatgatgatgatgttttgcagatatctca-3'), as previously described [26, 29]. Amplicons were cloned into a TOPO vector and the resulting plasmid was named pCRIL2. The number of nucleotides between the two ApaI sites flanking the whole insert (IL-2 ORF, GS, GE) is an exact multiple of six (504 bases).

\section{Construction of recombinant CDNA clones of rZJ1-IL2 and rZJ1-GFP}

The strategy for subcloning the modified IL-2 ORF from the pCRIL2 plasmid into the full-length clone of NDV strain ZJ1 (TVT7 expression vector) was previously described and was adopted in this study [26, 29]. The resulting full-length clone plasmid was designated pNDV/ZJ1-IL2.

\section{Rescue of the viruses}

Recombinant virus rZJ1-IL2 was rescued from pNDV/ ZJ1-IL2 plasmid in Hep-2 cells, as previously described $[26,40]$. From the allanotic fluid of infected eggs, RNA was extracted and the region corresponding to positions 2857-3676 of the NDV ZJ1 genome was amplified by RT-PCR and sequenced to confirm presence of the IL-2 insert. The other two viruses used in this study, rZJ1GFP and rZJ1 were previously plasmid-rescued and characterized [26]. The NDV strain rZJ1 is the parental strains, which was used to produce a full-length clone plasmid and to insert the green fluorescent protein (GFP) gene into the genome (rZJ1-GFP) [29]. The GFP gene is inserted in the same location of the IL-2 insert in rZJ1-IL2.

\section{Growth curves}

The replication kinetics of rZJ1-IL2, rZJ1-GFP, and rZJ1 were compared by multi-step growth curves in DF-1 and HD-11 cells. Cells were plated at $1 \times 10^{6}$ (DF-1) or $7 \times 10^{5}$ (HD-11) cells / well in 6 well plates and were infected with each NDV virus at a MOI $=0.01$ the following day. Briefly, maintenance media was discarded and cells were incubated with virus inoculum diluted in reduced-serum media ( $1 \%$ serum, $1 \mathrm{ml} /$ well) for $1 \mathrm{~h}$ at $37{ }^{\circ} \mathrm{C}$ with $5 \% \mathrm{CO}_{2}$. After adsorption, inoculum was 
removed, cells washed three times with PBS, and complete growth media (supplemented with $10 \%$ serum) was added. At $0,6,12,24,36,48$, and $72 \mathrm{~h}$ post infection (hpi), $200 \mu \mathrm{L}$ of supernatant were harvested for virus titration and replaced with $200 \mu \mathrm{L}$ of fresh media. Virus titers in the supernatant were determined by limiting dilution in DF-1 cells in 96-well plates and expressed as tissue culture infectious dose $50 \%\left(\mathrm{TCID}_{50}\right)$ per $\mathrm{ml}$ using the Spearman-Karber method. Each growth curve was conducted in triplicate.

\section{Eggs and chickens}

The Southeast Poultry Research Laboratory (SEPRL) specific pathogen free (SPF) White Leghorn flock was the source for all embryonated chicken eggs and chickens. All birds were housed in negative pressure isolators under biosafety level (BSL)-3 enhanced (E) conditions at SEPRL and provided food and water ad libitum. Virus propagation, isolation, and titration were conducted in nine to ten-day-old SPF embryonated eggs, by inoculation of infectious material in the chorioallantoic cavity [5]. Titration was carried out by limiting dilution in eggs, positive eggs were assessed by hemagglutination assay and results were expressed as embryo infectious dose 50 $\%\left(\mathrm{EID}_{50}\right)[5]$.

\section{Expression of IL-2 in vivo and in vitro}

The ability of rZJ1-IL2 to express chicken IL-2 was assessed by western blot (WB) in the supernatant and lysate of DF-1 cells, as well as in the allantoic fluid of embryonated eggs. DF-1 cells were plated at $5 \times 10^{5}$ cells/ well and infected the following day with rZJ1-GFP or rZJ1-IL2 at a MOI $=1$. After $48 \mathrm{~h}$, cell supernatant was collected and cells were harvested by adding Laemli buffer supplemented with $10 \% \beta$-Mercaptoethanol (lysis buffer) directly onto the cells. Allantoic fluid was extracted at $48 \mathrm{hpi}$ from eggs inoculated with $10^{3} \mathrm{EID}_{50}$ of rZJ1-IL2 or rZJ1-GFP in BHI media. Lysis buffer was mixed 1:1 with allantoic fluid or cell supernatant. All cell lysates were then incubated for $5 \mathrm{~min}$ at $100{ }^{\circ} \mathrm{C}$ and sonicated for $10 \mathrm{~s}$ at $70 \%$ amplitude with a cup sonicator (Branson, Dandury, CT). After separation in $12 \%$ SDSPAGE (pre-cast gels [Biorad, Hercules, CA]) at $150 \mathrm{~V}$, proteins were transferred by electrophoresis to a nitrocellulose membrane (semi-dry transfer, $30 \mathrm{~min}$ at $20 \mathrm{~V}$ ). After transfer, membranes were blocked for $1 \mathrm{~h}$ at room temperature (RT) with PBST (Phosphate-buffered saline, supplemented with $0.1 \%$ Tween) containing $5 \%$ bovine serum albumin (BSA). After blocking, primary antibodies for IL-2 (Polyclonal rabbit, Anti-chicken IL-2 [King Fisher, Carlsbad, CA]) and NDV nucleoprotein (in-house produced, hybridoma mouse monoclonal, clone 96-11) were diluted 1:3000 and 1:1000 respectively in blocking buffer and applied for $1 \mathrm{~h}$ at RT with constant shaking. Blots were then washed with PBST and incubated for $1 \mathrm{~h}$ at RT with secondary antibodies applied at 1:5000 dilutions (alkaline phosphatase-conjugated anti-rabbit or anti mouse [Santa Cruz, Dallas, TX]). Blots were then washed three times with PBST and incubated with chromogenic substrate (NBT/BCIP, Roche) until signal developed.

\section{Intracerebral Pathogenicity index test (ICPI)}

ICPI test was performed according to standard protocols $[3,4]$. Chickens were inoculated intracerebrally at oneday of age with $0.05 \mathrm{ml}$ of a 1:10 dilution of filtered infective allantoic fluid from rZJ1-IL2-infected eggs. Chickens were monitored daily and scored as normal, sick or paralyzed, and dead to compile a score over an 8 -day observation period. The final scores for each virus were tallied on a scale from 0 to 2 .

\section{Survival curves and clinical signs}

To assess the ability of rZJ1-IL2 and rZJ1-GFP to elicit clinical signs and cause death of infected birds, 4-weekold White Leghorn chickens were randomly assigned to seven experimental groups of 8-10 birds (rZJ1-IL2 groups at medium and high doses had 10 birds, the other groups had 8 birds), with each group allocated in a single isolator. Birds from each group were infected with a suspension of BHI containing either rZJ1-IL2 or rZJ1GFP at three different target doses: $10^{4}$ (low), $10^{5.5}$ (medium), and $10^{6.5}$ (high) $\mathrm{EID}_{50} /$ bird. Half of the inoculum $(0.05 \mathrm{ml})$ was delivered in the left conjunctival sac and the other half was delivered in the choanal slit. A BHI-inoculated group served as the negative control. Birds were observed for clinical signs every day and daily deaths for each group were recorded to tally a final survival curve for each virus at the given concentration. Birds that were in extremis at the time of observation were humanely euthanized and counted as dead for the next day.

\section{Pathogenesis assessment in chickens}

Sixty, 4-week-old SPF White Leghorn chickens were randomly allocated in three experimental groups of 20 birds each and inoculated with a BHI suspension of rZJ1-IL2, rZJ1-GFP, or BHI alone (mock-infected). The target dose of the inoculum was $10^{5.5} \mathrm{TCID}_{50}$ and it was applied with the same methods used for the survival curves. Birds were monitored daily for 5 days, and starting from day 1 post-infection (pi), 5 birds from each group were euthanized and spleens, blood, oropharyngeal and cloacal swabs were collected for virus isolation and titration. Additionally, the following tissues were harvested for histopathology from three out the five euthanized birds: eyelids, spleen, thymus, bursa of Fabricius, cecal tonsils, heart, brain, and liver. At each day, 
birds in extremis or presenting more pronounced clinical signs were sampled first for human reasons. Tissues were fixed in $10 \%$ neutral buffered formalin for $52 \mathrm{~h}$. All sampled tissues were routinely embedded in paraffin and $3 \mu \mathrm{m}$ sections were cut for hematoxylin and eosin staining (HE) and immunohistochemistry (IHC). The scoring system for assessment of microscopic lesions was previously published [41], and is reported in the caption of Table 2.

\section{Immunohistochemistry}

To detect viral distribution in tissues, IHC was performed on samples from rZJ1-IL2-, rZJ1-GFP, and mock-infected birds, as previously described [41]. Briefly, slides were deparaffinized, rehydrated, microwaved for 20 min at minimum power in Vector antigen unmasking solution (Vector Laboratories, Burlingame, CA) and blocked with an universal blocking reagent (Biogenex, San Ramon, CA). The primary antibody (polyclonal) was raised in rabbit against a synthetic peptide (TAYETADESETRRIC), which is highly conserved in NDV nucleoprotein, and used at 1:8000 dilution [42]. The detection system was an avidin-biotin-alkaline phosphatase system (Vector Laboratories, Burlingame, CA) coupled with a naphthol-based chromogen (Fast Red, Dako, Carpinteria, CA). Sections were counterstained lightly with hematoxylin and coverslipped with Permount for a permanent record. The scoring system for assessment of immunolabeling for NDV NP was previously published [41], and is reported in the caption of Table 2. For IL-2, the same procedure was deployed; the chicken IL-2 antibody was a rabbit polyclonal (Kingfisher, Carlsbad, CA) used at 1:1000 dilution.

\section{Virus isolation and titration}

Oral and cloacal swabs obtained from each bird were placed in separate tubes containing $1.5 \mathrm{ml}$ of brain-heart infusion broth (BHI) with antibiotics $(2000 \mathrm{U} / \mathrm{ml}$ penicillin $\mathrm{G}, 200 \mu \mathrm{g} / \mathrm{ml}$ gentamicin sulfate, and $4 \mu \mathrm{g} / \mathrm{ml}$ amphotericin B; Sigma Chemical Co., St. Louis, MO). Swab sample tubes were centrifuged at $1000 \times \mathrm{g}$ for $20 \mathrm{~min}$ and the supernatant removed for virus isolation and titration in eggs, according to standard procedures $[5,43]$.

Harvested spleens were homogenized with a Stomacher in a $10 \% \mathrm{w} / \mathrm{v}$ solution with PBS supplemented with antibiotics (2000 U/ml penicillin G, $200 \mu \mathrm{g} / \mathrm{ml}$ gentamicin sulfate, and $4 \mu \mathrm{g} / \mathrm{ml}$ amphotericin B [Sigma Chemical Co., St. Louis, MO]). For each sample, virus isolation was performed by inoculating undiluted homogenized samples into three embryonated eggs $(200 \mu \mathrm{L}$ per egg). Virus titer in positive eggs was assessed in embryonated eggs, as per standard procedures [5, 43].
Whole blood from chickens was harvested into EDTAcontaining tubes and $200 \mu \mathrm{L}$ were inoculated into three SPF embryonated eggs per sample for virus isolation (VI). Virus titers from VI-positive samples were subsequently assayed in embryonated eggs [5, 43].

\section{Data analysis}

Statistical analysis for growth curves of rZJ1-IL2 and rZJ1-GFP was carried out using a Two-Way ANOVA, followed by a Tukey test for multiple comparisons. A Log-Rank test with multiple comparison between groups was used for the survival curves. A non-parametric $t$-test (Mann-Whitney-Wilcoxon test) between rZJ1-IL2 and rZJ1-GFP groups was performed to assess the statistical differences in virus titers in spleen, blood, and swabs at the same time point. For each test, significance was considered with $p<0.05$. Statistical software was JMP version 8.0 (SAS Institute, Raleigh, NC). Survival curves were analyzed with GraphPad Prism (GraphPad Software, San Diego California USA).

\section{Additional file}

\begin{abstract}
Additional file 1: Table S1. Summary of clinical signs and mortality in 4-week-old chickens infected with rZJ1-GFP, rZJ1-IL2, and BHI. Numbers in the cells represent the percentages of birds presenting the clinical sign over the total birds present in the group at the time. Highlighted cells represent groups where all the animals are dead. (XLSX 13 kb)
\end{abstract}

\section{Abbreviations}

ND: Newcastle disease; NDV: Newcastle disease virus; IL-2: Interleukin-2; ICPI: Intracerebral pathogenicity index; WB: Western blot;

IHC: Immunohisotchemistry; HE: Hematoxylin and eosin; TCID50: Tissue culture infectious dose 50 \%; EID50: Embryo infectious dose 50 \%; SPF: Specific pathogen free; BHI broth: Brain-heart infusion broth.

\section{Competing interests}

The authors declare that they have no competing interests.

\section{Authors' contributions}

LS produced recombinant NDV strains, conducted growth curves, survival curves, histopathological and immunohistochemical analysis, statistical analysis, drafted the manuscript. DGD participated in titration of growth curve assays, titration of virus in organs and participated in pathogenesis experiments, reviewed the manuscript draft. SC conducted western blot and participated in pathogenesis experiments. SCG contributed to pathogenesis experiments (sample collection, titration). RSS provided plasmid containing chicken IL-2 and reagents for Western blot, critically reviewed and participated drafting the manuscript. PJM participated in study design, contributed to animal experiment (sample collection, titration) and manuscript revision. CCB contributed to study design, and manuscript drafting and revision. CLA conceived the study, participated in its design and coordination and helped to draft the manuscript. All authors read and approved the final manuscript.

\section{Acknowledgements}

We thank Tim Olivier and Jian Zhang for the excellent technical assistance.

Funding

This research was supported by USDA-ARS CRIS 6040-32000-064.

\section{Author details}

${ }^{1}$ USDA ARS, Southeast Poultry Research Laboratory, 934 College Station Rd, Athens, GA 30605, USA. ²Department of Veterinary Pathology, College of 
Veterinary Medicine, University of Georgia, Athens, GA 30605, USA. ${ }^{3}$ Department of Immunology and Microbiology, Wayne State University, Detroit, MI 48201, USA. ${ }^{4}$ Present address: Department of Pathobiology, Ontario Veterinary College, University of Guelph, Guelph, ON N1G 2W1, Canada. ${ }^{5}$ Present address: Department of Veterinary and Biomedical Sciences, College of Agriculture and Biological Sciences, South Dakota State University, Brookings, SD 57007, USA.

\section{Received: 25 May 2015 Accepted: 28 July 2015 \\ Published online: 08 August 2015}

\section{References}

1. Afonso C, Miller PJ. Newcastle disease: progress and gaps in the development of vaccines and diagnostic tools. In: Roth J, Ritch JA, Morozov IA, editors. Vaccines and diagnostics for transboundary animal diseases volume 135. Basel: Karger; 2013. p. 95-106.

2. Lamb R, Collins PL, Kolakofsky D, Melero JA, Nagay Y, Oldstone MBA, et al. The negative sense single stranded RNA viruses. In: Fauquet CM, Mayo MA Maniloff J, Desselberg U, Ball LA, editors. Virus taxonomy. San Diego, CA: Elsevier Academic Press; 2005. p. 607-738.

3. Miller PJ, Koch G, Suarez D, Mundt E, Jones RC, Rautenschlein S. Newcastle disease, other paramyxoviruses, and avian metapneumovirus infections. In: Swayne DE, Glisson JR, McDougald LR, Nolan LK, Suarez DL, Nair V, editors. Diseases of poultry. 13th ed. Ames, IA: Blackwell Publishing; 2013. p. 250-377.

4. OIE. World organization for animal health. Manual of diagnostic tests and vaccines for terrestrial animals. 6th ed. Paris, France: OIE; 2008. p. 576-89.

5. Alexander DJ, Senne DA. Newcastle disease and other avian paramyxoviruses. In: Dufour-Zavala L, Swayne DE, Glisson JR, Jackwood MW, Pearson JE, Reed WM, Woolcock PR, editors. A laboratory manual for the isolation, identification and characterization of avian pathogens. 4th ed. Athens, GA: American Association of Avian Pathologists; 2008. p. 135-41.

6. Cattoli G, Susta L, Terregino C, Brown C. Newcastle disease: a review of field recognition and current methods of laboratory detection. J Vet Diagn Invest. 2011:23:637-56.

7. Rasoli M, Yeap SK, Tan SW, Moeini H, Ideris A, Bejo MH, et al. Alteration in lymphocyte responses, cytokine and chemokine profiles in chickens infected with genotype VII and VIII velogenic Newcastle disease virus. Comp Immunol Microbiol Infect Dis. 2014;37:11-21.

8. Ecco R, Brown C, Susta L, Cagle C, Cornax I, Pantin-Jackwood M, et al. In vivo transcriptional cytokine responses and association with clinical and pathological outcomes in chickens infected with different Newcastle disease virus isolates using formalin-fixed paraffin-embedded samples. Vet Immunol Immunopathol. 2011:141:221-9.

9. Hu Z, Hu J, Hu S, Liu X, Wang X, Zhu J, et al. Strong innate immune response and cell death in chicken splenocytes infected with genotype VIld Newcastle disease virus. Virol J. 2012;9:208.

10. Rue CA, Susta L, Cornax I, Brown CC, Kapczynski DR, Suarez DL, et al. Virulent Newcastle disease virus elicits a strong innate immune response in chickens. J Gen Virol. 2011:92:931-9.

11. Tisoncik JR, Korth MJ, Simmons CP, Farrar J, Martin TR, Katze MG. Into the eye of the cytokine storm. Microbiol Mol Biol Rev. 2012;76:16-32.

12. Peiris JS, Cheung CY, Leung CY, Nicholls JM. Innate immune responses to influenza A H5N1: friend or foe? Trends Immunol. 2009;30:574-84.

13. Peiris JS, Hui KP, Yen HL. Host response to influenza virus: protection versus immunopathology. Curr Opin Immunol. 2010;22:475-81.

14. Stanford MM, McFadden G, Karupiah G, Chaudhri G. Immunopathogenesis of poxvirus infections: forecasting the impending storm. Immunol Cell Biol. 2007:85:93-102

15. Ghiasi H, Osorio Y, Perng GC, Nesburn AB, Wechsler SL. Overexpression of interleukin-2 by a recombinant herpes simplex virus type 1 attenuates pathogenicity and enhances antiviral immunity. J Virol. 2002;76:9069-78.

16. Ghiasi H, Cai S, Slanina SM, Perng GC, Nesburn AB, Wechsler SL. The role of interleukin (IL)-2 and IL-4 in herpes simplex virus type 1 ocular replication and eye disease. J Infect Dis. 1999;179:1086-93.

17. Reddehase MJ, Mutter W, Koszinowski UH. In vivo application of recombinant interleukin 2 in the immunotherapy of established cytomegalovirus infection. J Exp Med. 1987;165:650-6.

18. Hugin AW, Flexner C, Moss B. Clearance of recombinant vaccinia virus expressing IL-2: role of local host immune responses. Cell Immunol. 1993;152:499-509.
19. Perera LP, Goldman CK, Waldmann TA. Comparative assessment of virulence of recombinant vaccinia viruses expressing $\mathrm{IL}-2$ and IL-15 in immunodeficient mice Proc Natl Acad Sci U S A. 2001;98:5146-51.

20. Bukreyev A, Whitehead SS, Prussin C, Murphy BR, Collins PL. Effect of coexpression of interleukin-2 by recombinant respiratory syncytial virus on virus replication, immunogenicity, and production of other cytokines. J Virol. 2000;74:7151-7.

21. Kinter AL, Bende SM, Hardy EC, Jackson R, Fauci AS. Interleukin 2 induces CD8+ T cell-mediated suppression of human immunodeficiency virus replication in CD4+ T cells and this effect overrides its ability to stimulate virus expression. Proc Natl Acad Sci U S A. 1995:92:10985-9.

22. Sundick RS, Gill-Dixon C. A cloned chicken lymphokine homologous to both mammalian IL-2 and IL-15. J Immunol. 1997:159:720-5.

23. Kaiser $P$, Mariani P. Promoter sequence, exon:intron structure, and synteny of genetic location show that a chicken cytokine with T-cell proliferative activity is IL2 and not IL15. Immunogenetics. 1999;49:26-35.

24. Stepaniak JA, Shuster JE, Hu W, Sundick RS. Production and in vitro characterization of recombinant chicken interleukin-2. J Interferon Cytokine Res. 1999;19:515-26.

25. Staeheli P, Puehler F, Schneider K, Gobel TW, Kaspers B. Cytokines of birds: conserved functions-a largely different look. J Interferon Cytokine Res. 2001:21:993-1010.

26. Susta L, Cornax I, Diel DG, Garcia SC, Miller PJ, Liu X, et al. Expression of interferon gamma by a highly virulent strain of Newcastle disease virus decreases its pathogenicity in chickens. Microb Pathog. 2013;61-62:73-83.

27. Kho CL, Tan WS, Tey BT, Yusoff K. Newcastle disease virus nucleocapsid protein: self-assembly and length-determination domains. J Gen Virol. 2003:84:2163-8.

28. Hierholzer JC, Killington RA. Virus isolation and quantification. In: Mahy WJB, Kangro HO, editors. Virology methods manual. London: Academic Press Limited; 1996. p. 37-9.

29. Liu YL, Hu SL, Zhang YM, Sun SJ, Romer-Oberdorfer A, Veits J, et al. Generation of a velogenic Newcastle disease virus from CDNA and expression of the green fluorescent protein. Arch Virol. 2007;152:1241-9.

30. Krishnamurthy S, Huang Z, Samal SK. Recovery of a virulent strain of newcastle disease virus from cloned cDNA: expression of a foreign gene results in growth retardation and attenuation. Virology. 2000;278(1):168-82

31. Liao W, Lin JX, Leonard WJ. Interleukin-2 at the crossroads of effector responses, tolerance, and immunotherapy. Immunity. 2013;38:13-25.

32. Blattman JN, Grayson JM, Wherry EJ, Kaech SM, Smith KA, Ahmed R. Therapeutic use of IL-2 to enhance antiviral T-cell responses in vivo. Nat Med. 2003:9:540-7.

33. Straub C, Neulen ML, Sperling B, Windau K, Zechmann M, Jansen CA, et al. Chicken NK cell receptors. Dev Comp Immunol. 2013;41:324-33.

34. Selvaraj RK. Avian CD4(+)CD25(+) regulatory T cells: properties and therapeutic applications. Dev Comp Immunol. 2013;41:397-402.

35. Guo P, Thomas JD, Bruce MP, Hinton TM, Bean AG, Lowenthal JW. The chicken TH1 response: potential therapeutic applications of ChIFN-gamma. Dev Comp Immunol. 2013:41:389-96.

36. Erf GF. Cell-mediated immunity in poultry. Poult Sci. 2004;83:580-90.

37. Kapczynski DR, Afonso CL, Miller PJ. Immune responses of poultry to Newcastle disease virus. Dev Comp Immunol. 2013;41:447-53.

38. Puddu P, Carollo M, Pietraforte I, Spadaro F, Tombesi M, Ramoni C, et al. IL-2 induces expression and secretion of IFN-gamma in murine peritoneal macrophages. J Leukoc Biol. 2005;78(3):686-95.

39. Beug H, von Kirchbach A, Doderlein G, Conscience JF, Graf T. Chicken hematopoietic cells transformed by seven strains of defective avian leukemia viruses display three distinct phenotypes of differentiation. Cell. 1979:18:375-90.

40. Estevez C, King D, Seal B, Yu Q. Evaluation of Newcastle disease virus chimeras expressing the Hemagglutinin-Neuraminidase protein of velogenic strains in the context of a mesogenic recombinant virus backbone. Virus Res. 2007;129:182-90.

41. Susta L, Jones ME, Cattoli G, Cardenas-Garcia S, Miller PJ, Brown CC, et al, Pathologic characterization of genotypes XIV and XVII Newcastle disease viruses and efficacy of classical vaccination on specific pathogen-free birds. Vet Pathol. 2015;52:120-31.

42. Kommers GD, King DJ, Seal BS, Brown CC. Virulence of pigeon-origin Newcastle disease virus isolates for domestic chickens. Avian Dis. 2001:45:906-21.

43. McGinnes LW, Pantua H, Reitter J, Morrison TG. Newcastle disease virus: propagation, quantification, and storage. Curr Protoc Microbiol. 2006; Chapter 15:Unit 15 F 12. doi: 10.1002/9780471729259.mc15f02s01. 